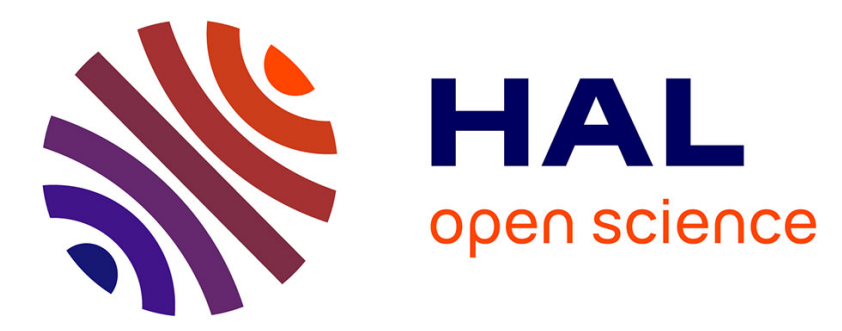

\title{
New spider mites (Acari: Tetranychidae) of the genera Paraplonobia and Eurytetranychus from Iran, and a description of all life stages of Eutetranychus orientalis (Klein)
}

\author{
Masoumeh Khanjani, Mohammad Khanjani, Owen D. Seeman
}

\section{To cite this version:}

Masoumeh Khanjani, Mohammad Khanjani, Owen D. Seeman. New spider mites (Acari: Tetranychidae) of the genera Paraplonobia and Eurytetranychus from Iran, and a description of all life stages of Eutetranychus orientalis (Klein). Acarologia, 2017, 57 (3), pp.465-491. 10.24349/acarologia/20174167 . hal-01535581

\section{HAL Id: hal-01535581 \\ https://hal.science/hal-01535581}

Submitted on 9 Jun 2017

HAL is a multi-disciplinary open access archive for the deposit and dissemination of scientific research documents, whether they are published or not. The documents may come from teaching and research institutions in France or abroad, or from public or private research centers.
L'archive ouverte pluridisciplinaire $\mathbf{H A L}$, est destinée au dépôt et à la diffusion de documents scientifiques de niveau recherche, publiés ou non, émanant des établissements d'enseignement et de recherche français ou étrangers, des laboratoires publics ou privés.

\section{(ㅇ)(1) $\$$}

Distributed under a Creative Commons Attribution - NonCommercial - NoDerivatives| 4.0 


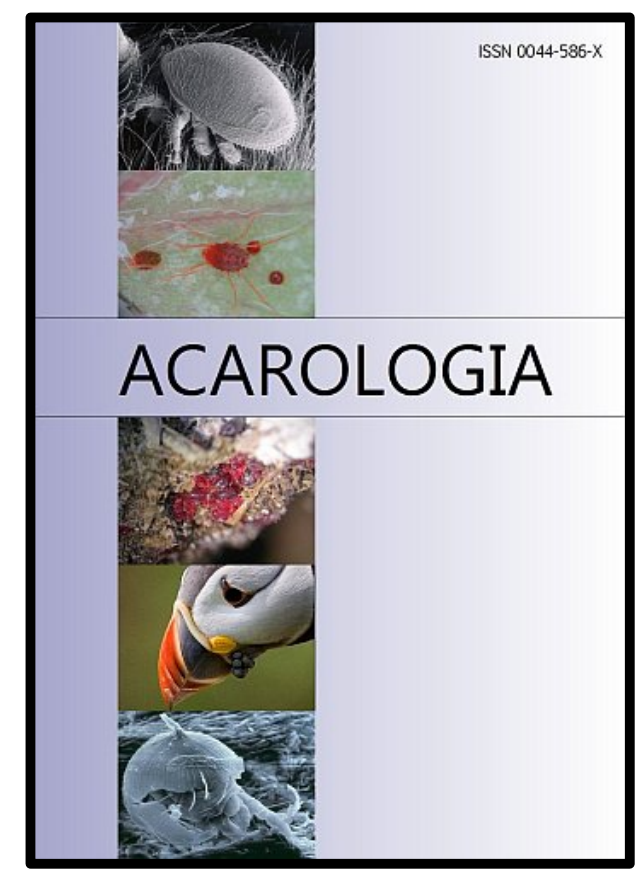

\section{ACAROLOGIA}

A quarterly journal of acarology, since 1959

Publishing on all aspects of the Acari

All information:

http://www1.montpellier.inra.fr/CBGP/acarologia/ acarologia@supagro.inra.fr

\section{OPEN ACCESS}

\section{Acarologia is proudly non-profit, with no page charges and free open access}

Please help us maintain this system by encouraging your institutes to subscribe to the print version of the journal and by sending us your high quality research on the Acari.

Subscriptions: Year 2017 (Volume 57): $380 €$ http://www1.montpellier.inra.fr/CBGP/acarologia/subscribe.php

Previous volumes (2010-2015): $250 € /$ year (4 issues)

Acarologia, CBGP, CS 30016, 34988 MONTFERRIER-sur-LEZ Cedex, France

The digitalization of Acarologia papers prior to 2000 was supported by Agropolis Fondation under the reference ID 1500-024 through the « Investissements d'avenir » programme

(Labex Agro: ANR-10-LABX-0001-01)
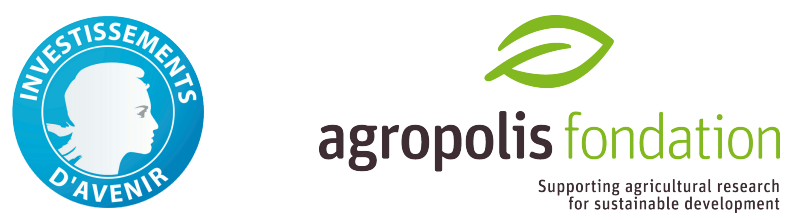

Acarologia is under free license and distributed under the terms of the

Creative Commons-BY-NC-ND which permits unrestricted non-commercial use, distribution, and reproduction in any medium, provided the original author and source are credited. 


\title{
New spider mites (Acari: Tetranychidae) of the genera Paraplonobia and Eurytetranychus from Iran, and a description of all life stages of Eutetranychus orientalis (Klein)
}

\author{
Masoumeh KHANJANI ${ }^{1}$, Mohammad KHANJANI ${ }^{1}{ }^{1}$ and Owen D. SEEMAN ${ }^{2}$ \\ (Received 28 August 2016; accepted 01 November 2016; published online 15 May 2017; edited by Philippe AUGER) \\ ${ }^{1}$ Department of Plant Protection, College of Agriculture, Bu - Ali Sina University, Hamedan, Iran. mh.khanjani86@gmail.com, \\ mkhanjani@gmail.com $(\bowtie)$ \\ 2 Queensland Museum, PO Box 3300, South Brisbane, Qld, 4101, South Brisbane, Australia.owen.seeman@qm.qld.gov.au
}

\begin{abstract}
Two new species of Paraplonobia Wainstein and Eurytetranychus Oudemans are described from Iran: Paraplonobia (Paraplonobia) flechtmanni n. sp. from Russian knapweed Rhaponticum repens (Asteraceae) and Eurytetranychus aminii n. sp. from gum bushes Astragalus gossypinus (Fabaceae). We describe all life stages of Eutetranychus orientalis (Klein) and discuss variation within this polyphagous pest species. A key to all known species of the genus Paraplonobia (Paraplonobia) is given.
\end{abstract}

KEYWORDS - Tetranychoidea; taxonomy; key; Eurytetranychini; Hystrichonychini; plant

ZOOBANK — 65B35917-251F-41E0-857D-ED80441BAFDE

\section{INTRODUCTION}

The spider mites (Acariformes: Tetranychidae) are the largest and most important family of the super family Tetranychoidea, comprising 1,288 species (Migeon \& Dorkeld 2006-2015) and numerous pest species (Jeppson et al. 1975; Baker \& Tuttle 1994). Its subfamily, tribe and genus level classification have been built, and modified, by several significant works such as Pritchard \& Baker (1955), Wainstein (1960), Meyer (1974, 1987), and Gutierrez (1985). The classification presented in Bolland et al. (1998) is generally followed, with the family classified into two subfamilies each split into three tribes (Bryobiinae: Bryobiini, Hystrichonychini, Petrobiini; Tetranychinae: Eurytetranychini, Tenuipalpoi- dini, Tetranychini). Although of practical value, this classification is not necessarily stable. For example, Hernandes \& Feres (2013) reinstated the tribe Aponychini (Tetranychinae) from three Eurytetranychine genera.

In this work we describe a new species of Paraplonobia Wainstein, 1960 (Hystrichonychini), a new species of Eurytetranychus (Eurytetranychini) and redescribe the polymorphic species Eutetranychus orientalis (Eurytetranychini) based on all active life stages of the Iranian specimens. This is the first species of Paraplonobia recorded from Iran, although another unidentified species was previously reported by Khanjani \& Kamali (1998). The genus Eurytetranychus is represented by two species 
in Iran, Eur. admes Pritchard \& Baker and Eur. buxi (Garman), while Eutetranychus is known from five species: Eut. africanus (Tucker), Eut. banksi (McGregor), Eut. fici Meyer, Eut. orientalis (Klein) and Eut. palmatus Attiah (Beyzavi et al. 2013). Eutetranychus orientalis is a widespread species found on numerous host plants, on which it is sometimes a pest (Jeppson et al. 1975). The species is polymorphic, varying especially in the form of its dorsal setae (Meyer 1987), and several species are already considered synonyms, which are: Anychus ricini Rahman and Sapra, 1940; Eutetranychus monodi André, 1954; Eut. sudanicus El Badry, 1970; Eut. Anneckei Meyer, 1974 (see Bolland et al. 1998). In Iran, it is known from numerous localities and host plants (Beyzavi et al. 2013).

\section{MATERIALS AND METHODS}

Leaves of plants infested by spider mites were taken to the laboratory for processing. Mites were removed from leaves with a No. 0 paint brush under a stereomicroscope and mounted in Hoyer's medium. The specimens were measured and drawn by using differential interference contrast microscopy at $1000 \times$ magnification on an Olympus BX 51 (Iran) or Nikon Eclipse 80i (Australia) microscope.

Body length measurements represent the distance between tip of gnathosoma to end of idiosoma; width was measured above coxae III; setae were measured from their insertion to their tips; distance between setae was measured as the distance between their insertions. Legs were measured from the base of the trochanter to the pretarsus (base of claws).

The terminology, abbreviations and leg chaetotaxy used in the descriptions follows those of Lindquist (1985). Leg setal counts are presented as "total number of tactile setae and eupathidia (total number of solenidia+number of duplex pairs)". All measurements are given in micrometers and the measurements of the paratypes are given in parentheses after the measurement for the holotype.

\section{TAXONOMY}

\section{Subfamily Bryobiinae Berlese \\ Tribe Hystrichonychini Pritchard and Baker Genus Paraplonobia Wainstein}

Aplonobia (Paraplonobia) Wainstein, 1960: 140.

Paraplonobia (Paraplonobia), Tuttle and Baker, 1968:50.

Paraplonobia, Meyer, 1974: 119; Tuttle and Baker, 1968:48; Tuttle, Baker \& Abbatiello, 1976:19; Baker and Tuttle, 1994:83.

Type species: Aplonobia (Paraplonobia) echinopsili Wainstein, by original designation.

Diagnosis - With the features of the Hystrichonychini plus the following. Three pairs of prodorsal setae ( $v 1$ absent); prodorsum without lobes; ten pairs of dorsal opisthosomal setae (c1-3, d1-2, e1-2, $f 1-2, h 1$, note two pairs of $e$ and two pairs of $f$ setae present, $d 3$ and $e 3$ absent); seta $f 2$ in dorso-sublateral position (never lateral); body not extremely elongate, usually no more than twice as long as wide ( $P$. boutelouae slightly greater than $2 \times$ ); opisthosoma without plates or strong tubercles (weak tubercles in P. echinopsili); coxal and coxisternal setal formula usually hypotrichous, not exceeding holotrichous condition of 4-3-2-2; tarsus I with two sets of duplex setae. Claw and empodium pad like.

Remarks - Paraplonobia was established as a subgenus of Aplonobia by Wainstein (1960). The subgenus was later raised to genus by Tuttle \& Baker (1968), although their keys to genera and their generic definition of Paraplonobia conflicted. While the key (couplet 17) split the genus Paraplonobia (simple peritremes) from Anaplonobia and Neopetrobia (anastomosing peritremes), their subgenera of Paraplonobia had simple or anastomosing peritremes. Those Paraplonobia with anastomosing peritremes were the subgenus Langella Wainstein, demoted to subgeneric rank by Tuttle \& Baker (1968). Meyer (1974) recognized this problem and removed Langella from Paraplonobia, returning Langella to generic rank. She provided an updated diagnosis and considered Paraplonobia to include four species: P. echinopsili (Wainstein), P. hilariae Tuttle \& Baker, P. myops (Pritchard \& Baker) and P. tridens Tuttle \& Baker. 
Gutierrez (1985) and Meyer (1987) then independently modified the definition of Paraplonobia and its related genera. Gutierrez (1985) formed a broader definition of Paraplonobia by including under it genera with anastomosing peritremes (Anaplonobia) and holotrichous coxal setation (the monotypic Brachynychus), thereby demoting these two genera to subgenera. In contrast, Meyer (1987) was more restrictive, and considered Anaplonobia and Brachynychus as separate genera. Meyer's (1987) definition of Brachynychus was similar to that of Gutierrez (1985), except she also noted that this species has empodial pads and true claws that are equal in length and the claws bear only one pair of tenent hairs (as opposed to the claws being longer and bearing two pairs of tenent hairs). Meyer's (1987) definition of Anaplonobia acknowledged its anastomosing peritremes, but also noted that tarsus I lacks the tectal setae and that the coxal formula is 2-3-1-1 or 2-2-1-1.

Due to widespread use of Gutierrez's (1985) classification, we follow Gutierrez (1985) here. Our genus and subgenus diagnoses are essentially derived from Gutierrez (1985). This definition of Gutierrez (1985) is used by Bolland et al. (1998) and Migeon \& Dorkeld (2006-2015) to create the current membership of Paraplonobia: Paraplonobia (Anaplonobia) - 28 species; Paraplonobia (Brachynychus) - 1 species; and Paraplonobia (Paraplonobia) - 10 species.

\section{Subgenus Paraplonobia Wainstein}

Diagnosis - With the features of Paraplonobia plus the following: coxal setal formula 2-2-1-1 (i.e. 1b, 1c, $2 b, 2 c, 3 b, 4 b)$; peritremes simple. Anal plate with three pairs of setae, tarsus II with one duplex seta (Smiley \& Baker, 1995).

\section{Paraplonobia (Paraplonobia) flechtmanni n. sp. (Figures. 1-2)}

\section{Zoobank: 8D3CE505-86CB-40AE-B441-F68D5DEF4A01}

Diagnosis - Prodorsal shield demarcated by longitudinal, broken striae; dorsal body setae slender, pointed distally, moderately long: setae $c 1$ extending past bases of $d 1$, setae $f 1$ extending past bases of $h 1$; other dorsal setae reaching at least halfway to corresponding seta in next row. Stylophore slightly cleft anteriorly. Leg I almost as long as or slightly longer than body; tarsi III and IV with a duplex setal complex; femora 9-6-4-4; genua 5-5-4-

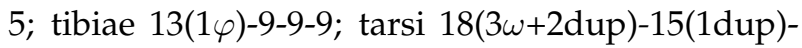
13(1dup)-13(1dup).

Description. Female - (Figs. 1-2; n=2). Idiosoma elongate. Body length including gnathosoma 608(550), excluding gnathosoma 456(450); width 335 (343).

Dorsum (Fig. 1A) - Median propodosomal shield with broken, longitudinal striae; lateral idiosoma with longitudinal to oblique striae; opisthosoma with transverse striae medially and posteriorly (Fig. 1A). Dorsal setae slender, laterally serrate and pointed distally. Lengths of dorsal setae $v 2$ 62(62), sc1 45(40), sc2 52(46), c1 54(52), $c_{2}$ 52(48), c $355(52), d 1$ 42(47), d2 56(53), e1 49(50), e2 62(59), f1 71(72), f2 66(68), h1 65(65). Distances between dorsal setae; v2-h1 406(380), v2-v2 67(65), sc1sc1 165(167), sc2-sc2 235(237), c1-c1 128(133), d1-d1 104(95), e1-e1 64(63), f1-f1 42(38), h1-h1 41(36), c1-d1 34(46), d1-e1 75(85), e1-f1 64(74), f1-h1 55(56).

Venter (Figs. 1B, C) - Coxisternal area between coxae I-III and behind coxa IV with transverse striae; area between setae $3 a-4 a$ with longitudinal striae and posterior opisthosoma with longitudinal to oblique striae (Fig. 1B). Lengths of setae $1 a$ 47(51), $1 b$ 52(54), 1c 38(37), $2 b$ 37(39), 2c 33(34), $3 a$ 36(39), $3 b$ 38(43), 4a 53(50), $4 b$ 46(48), ag 63(56), g1 57(60), g2 39(33), ps1 17(21), $p s_{2}$ 15(24), $p s_{3} 19(23)$, h2 22(22), h3 27(30).

Gnathosoma (Figs. 1B, 1D-F) - Ventral infracapitulum with two pairs of adoral setae or $_{1} 5$ (5) and or $_{2} 6$ (5-6) and one subcapitular seta $m$ 37(35). Palp eupathidion $s u \xi$ length 6(6), width 1(1), solenidion $\omega$ length 3(3), width 2(2) (Fig. 1E). Stylophore slightly emarginated anteriorly (Fig. 1D). Peritremes simple and oval-shaped distally (Fig. 1F).

Legs (Figs. 2A-E) - Leg I 626(570); leg II 350(329); leg III 379(366), leg IV 485(452). Leg I almost as long as or slightly longer than body length. Leg segment setal formula as follows: coxae 2-2-11; trochanters 1-1-1-1; femora 9-6-4-4; genua 5-5-4-5;

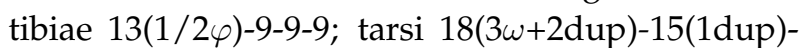
13(1dup)-13(1dup). Tarsus I and III with solenidia 

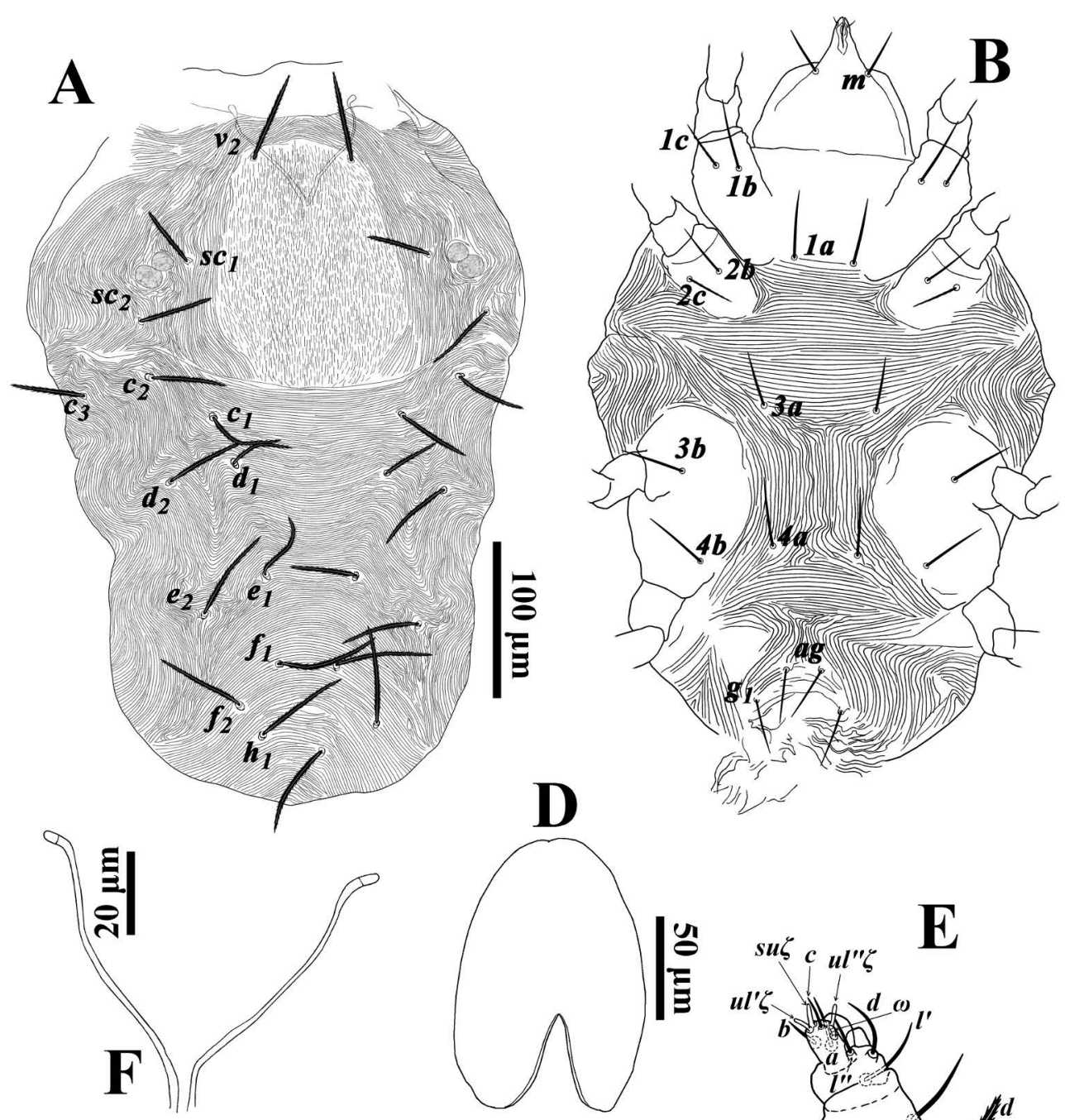

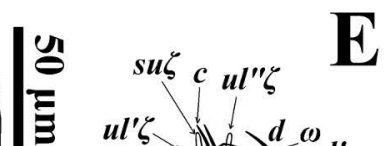
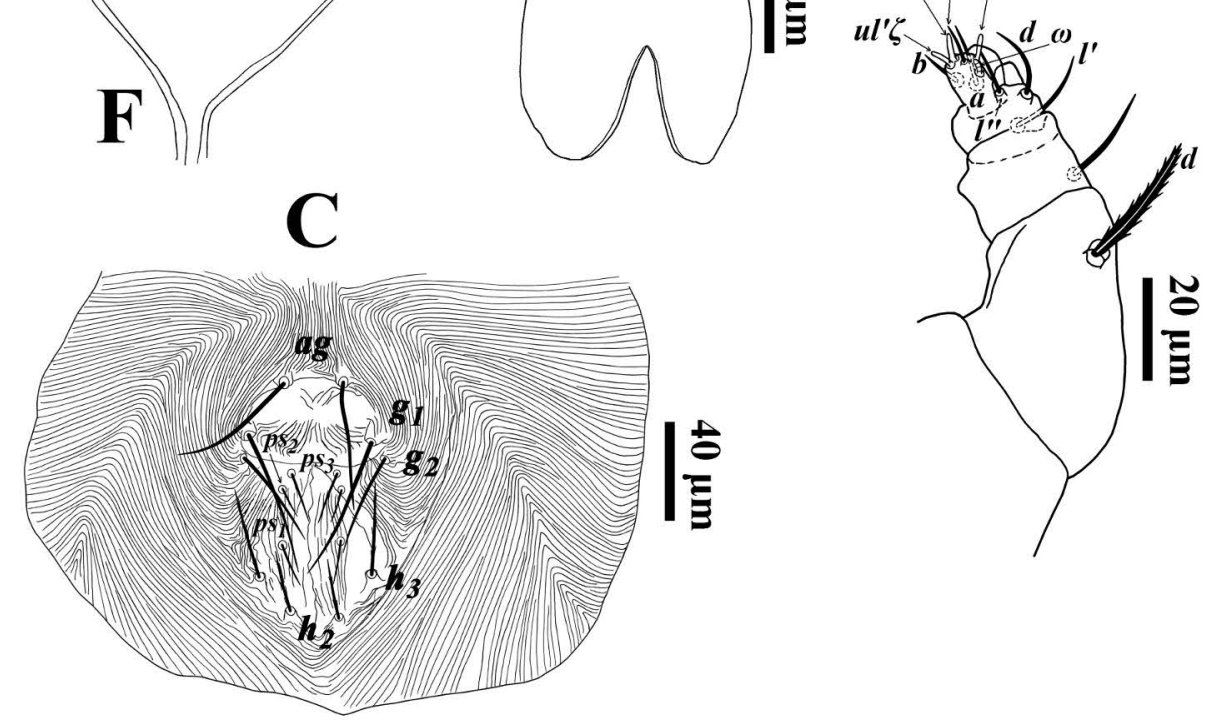

FIgURE 1: Paraplonobia flechtmanni n. sp. Female: A - Dorsal view; B - Ventral view; C - Genitoanal area; D - Stylophore; E - Palp; F Peritrems. 


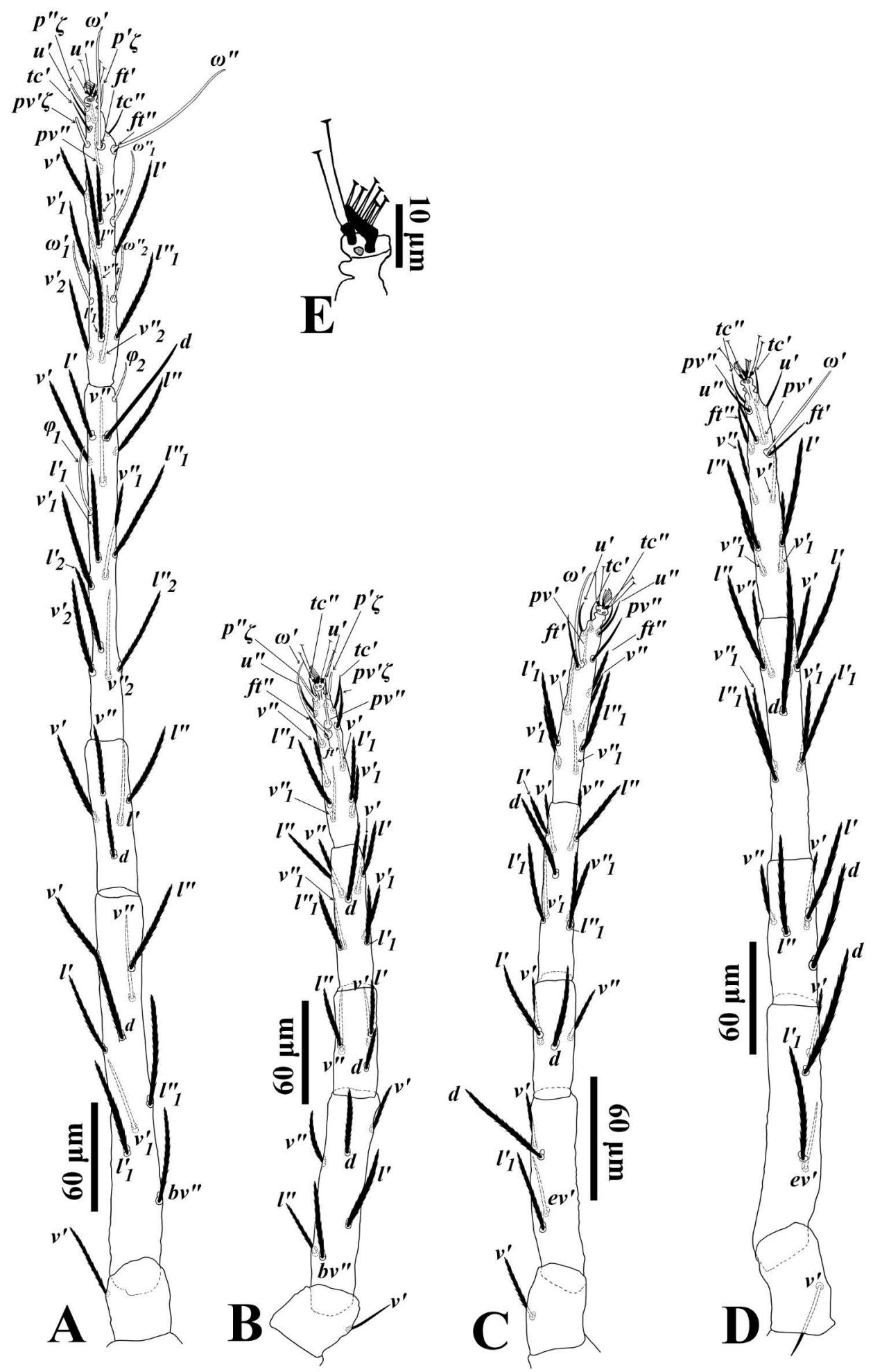

FIGURE 2: Paraplonobia flechtmanni n. sp. Female: A - Leg I; B - Leg II; C - Leg III; D - Leg IV; E - Empodium and claws. 
$\mathrm{I} \omega^{\prime} 1$ 33(29), I $\omega^{\prime \prime} 1$ 41(42), I $\omega^{\prime \prime} 2$ 32(32), I $\omega^{\prime}$ 63(73), I $\omega^{\prime \prime}$ 73(76), II $\omega^{\prime} 49(51)$, III $\omega^{\prime}$ 52(50), IV $\omega^{\prime}$ 52(55) and tibia I with I $\varphi 1$ 21(18). Leg setae subulate (Figs. 2AD). Claw and empodium padlike (Fig. 2E).

Material examined - Holotype female and one paratype female, ex. Russian knapweed, Rhaponticum repens (L.) Hidalgo (Asteraceae), Iran: Hamedan province, Hamedan, Neshar village,

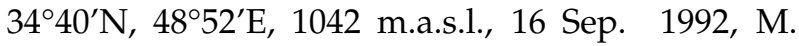
Khanjani. The holotype female is deposited as slide-mounted specimens in the Collection of the Acarology Laboratory, University of Bu-Ali Sina, Hamadan, Iran and the paratype female will be deposited in the Queensland Museum, South Brisbane, Australia.

Etymology - This species is named in honor of Prof. Dr. Carlos Flechtmann, Departamento de Entolomogia e Acarologia (LEA) (ESALQ), University of São Paulo, São Paulo, Brazil, for his great contribution to the development of Acarology in the world.

Remarks - The new species Paraplonobia flechtmanni n. sp. is comparable with other Paraplonobia with legs about as long as or slightly longer than the body, i.e., P. hilariae Tuttle \& Baker, 1968, P. tridens Tuttle \& Baker, 1968 and P. myops (Pritchard \& Baker, 1955). Of these species, P. flechtmanni n. sp. resembles $P$. hilariae most closely in having a slightly cleft stylophore and long, slender, pointed dorsal setae. However it differs from $P$. hilariae in: prodorsum with longitudinal, broken striae medially instead of longitudinal striae in $P$. hilariae; setae $c 1$ extending past the bases of $d 1$ compared with not extending to bases of $d 1$ in $P$. hilariae; tarsi III-IV with duplex setae versus without duplex setae in P. hilariae; and the solenidia on tarsi III-IV are as long as or longer than the tarsal setae, compared with being shorter than other tarsal setae in P. hilariae. The presence of duplex setae on leg III-IV in our new species is not the addition of a seta, but instead is the close association of the anterior fastigial seta with the solenidion. This is confirmed by the examination of females of an undescribed Australian Paraplonobia that has the same tarsal III-IV setation $(14+1 \omega)$ but lack duplex setae.

\section{Key to species of Paraplonobia (Paraplonobia) (after Meyer 1987, based on literature)}

1. Stylophore rounded anteriorly $\ldots \ldots \ldots \ldots \ldots 2$

— Stylophore cleft anteriorly ................ 3

2. Dorsal body setae lanceolate; leg I shorter than body ................. P. edenvillensis Meyer — Dorsal body setae slender; leg I about as long as body............. myops (Pritchard and Baker)

3. Dorsal body setae slender and pointed distally . 4 — Dorsal body setae broadly lanceolate.........7

4. Leg I as long as or slightly longer than body . . 5 - Leg I shorter than body ............... 6

5. Prodorsum with longitudinal striae; tarsi III and IV with a distal slender solenidion................ P. hilariae Tuttle and Baker - Prodorsum with longitudinal broken striae; tarsi III and IV with a duplex setae..................... Plechtmanni n. sp.

6. First three pairs of dorsocentral setae $(c 1, d 1$, e1) about half as long as distances between consecutive setae; posterior opisthosomal setae $(f 1, f 2)$ shorter than longitudinal distances between their bases............... Perniaria (Bagdasarian)

- First three pairs of dorsocentral setae $(c 1, d 1$, e1) minute, about a third to fourth as long as distances between bases of consecutive setae; posterior opisthosomal setae $(f 1, f 2)$ longer than longitudinal distances between their bases.

P. boutelouna Baker and Tuttle

7. Dorsocentral setae $(c 1, d 1, e 1$ and $f 1)$ more than half as long as distances between consecutive setae.................................. 9

- Dorsocentral setae ( $c 1, d 1, e 1$ and $f 1$ ) almost half or one third as long as distances between consecutive setae.......................... 8

8. Prodorsum with small punctuations; ratio e1/e1-e1 0.78; leg I slightly longer than 
body................. P. tridens Tuttle and Baker - Prodorsum with longitudinal striae; ratio $e 1 / e 1$ e1 0.39; leg I shorter than body

P. dactyloni Smiley and Baker

9. Peritremal ending rounded; prodorsum with a well-marked punctate shield; tibia IV with 8 setae................... P. penicillatus Chaudhri - Peritremal ending oval; prodorsum without a well-marked punctate shield; tibia IV with 7 setae.................. P. echinopsili (Wainstein)

\section{Subfamily Tetranychinae Tribe Eurytetranychini Reck Genus Eutetranychus Banks}

Neotetranychus (Eutetranychus) Banks, 1917:197. Anychus McGregor, 1919: 644.

Type species: Tetranychus banksi McGregor, by original designation.

Diagnosis - Dorsal opisthosoma with ten pairs of setae (c1-3, d1-2, e1-2, f1-2, h1), venter with setae h2-3; dorsal setae may be inserted on tubercles; peritremes simple or slightly expanded distally; anal valves with two pairs of anal setae (ps1-2); tarsi I and II each bear a pair of 'associated setae' (bases of $f t^{\prime \prime}$ and $\omega^{\prime \prime}$ touching, or nearly so; not coalesced), true claws pad-like, with tenent hairs, empodium absent or a rudimentary knob.

\section{Eutetranychus orientalis (Klein) (Figures 3-9)}

Diagnosis — Based partially on Meyer (1987). Female: propodosoma with longitudinal striae and with distinct strial lobes; opisthosomal striae transverse, except between setae d1-e1 longitudinal to forming a V- or U- pattern; setae $c 2, c 3, d 2, e 2, f 2$ variable in length and form, but longer than setae $c 1, d 1, e 1, f 1, h 1$, which are often short; dorsal setae plumose, thickened, but otherwise variable in form: dorsocentral setae $c 1, d 1, e 1, f 1, h 1$ tending to be lanceolate, ovate or obovate, and dorsolateral setae and sublateral seta $c 2$ subulate, oblanceolate or spatulate (but sometimes dorsocentral setae taking form of dorsolateral setae, see Chaudhri et al. 1974); dorsal setae inserted on small tubercles; distance c1$c 1$ and f1-f1 subequal; spermatheca elongated and oval, sometimes with pointed tip; coxae II with one seta; leg setal counts subject to some intraspecific variation, but typically as follows: femora 8-6-3-1; genua 5-5-2-2; tibiae $9(1 \varphi)-6-6-7$; tarsi $15(3 \omega)-13(1 \omega)$ $10(1 \omega)-10(1 \omega)$. Male: distal bent portion of aedeagus shorter than dorsal margin of shaft.

Description - Female (Figs. 3-4; $\mathrm{n}=156$ ) Color in life red. Idiosoma broad oval. Body length including gnathosoma 382-462, excluding gnathosoma 292-385; width 275-317.

Dorsum - (Figs. 3A-B). Prodorsum medially with longitudinal striae and with distinct strial lobes (Fig. 3B); area between setae $c 1-d 1$ and $e 1$ $h 1$ with transverse striae, striae between setae d1$e 1$ form a V- pattern, idiosoma with longitudinal to oblique striae laterally; dorsal striae with minute knobs (Fig. 3A). Dorsal setae inserted on small tubercles and vary in length and shape, propodosomal and dorsolateral setae and setae $c 2, h 1$ long and oblanceolate-spatulate, dorsocentral setae $(c 1, d 1, e 1$ and f1) short and oblanceolate. Lengths of dorsal setae: v2 41-54, sc1 33-57, sc2 41-54, c1 15-31, $c_{2}$ 40-58, $c_{3} 42-51, d 1$ 19-30, d2 39-58, e1 18-28, e2 37-54, f1 20$36, f 2$ 37-50, h1 27-41. Distances between dorsal setae: v2-h1 287-369, v2-v2 49-60, sc1-sc1 109-128, sc2sc2 193-226, c1-c1 48-62, d1-d1 98-122, e1-e1 40-59, f1f1 44-52, h1-h1 25-41, c1-d1 38-66, d1-e1 40-82, e1-f1 25-55, f1- $h 1$ 46-87.

Venter - (Figs. 3C-F). Coxisternal area and behind coxa IV with transverse striae; posterior opisthosoma with longitudinal to oblique striae in an inverse U-shape. Lengths of setae: $1 a$ 40-49, $1 b$ 47-57, $1 c$ 49-58, $2 b$ 46-56, 3a 45-52, 3b 47-60, 4a 4254, $4 b$ 46-58, ag 42-56, g1 31-39, g2 30-35, ps1 10$16, p s_{2}$ 11-16, h2 17-26, $h 3$ 17-25. Spermatheca oval and elongated, and saclus terminally rounded or pointed (Figs. 3E-F).

Gnathosoma (Figs. 3C, G) - Ventral infracapitulum with two pairs of adoral setae and one subcapitular seta $m$ 38-52. Palp eupathidion $s u \xi$ length 7-9, width 2, solenidion $\omega$ length $4-5$, width 1 . Stylophore anteriorly rounded; peritremes with simple bulb distally (Fig. 3A). 

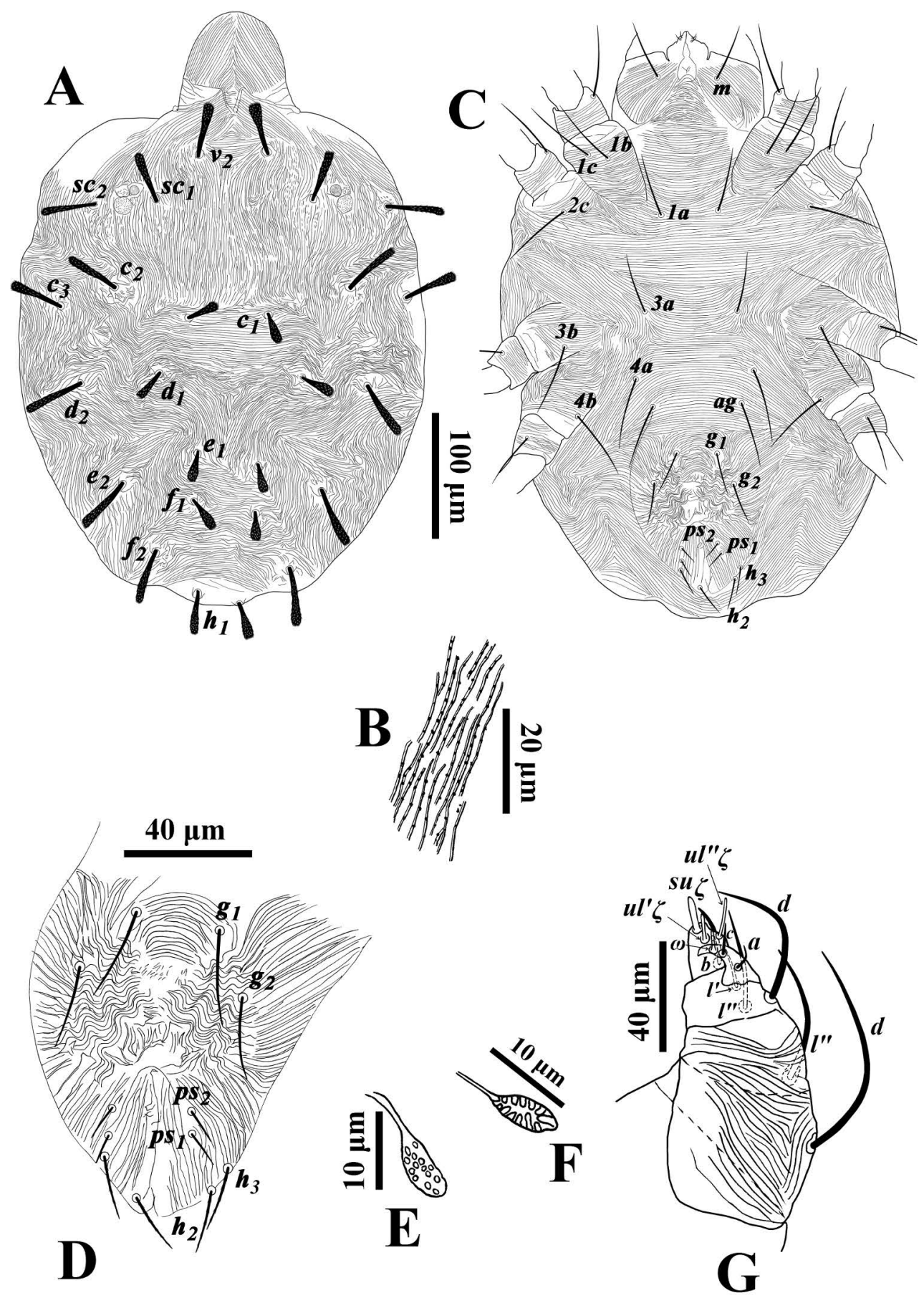

FIGURE 3: Eutetranychus orientalis (Klein) Female: A - Dorsal view; B - Dorsal pattern; C - Ventral view; D - Genital area; E - Spermatheca (Rounded at tip); F - Spermatheca (Pointed at tip); G - Palp. 


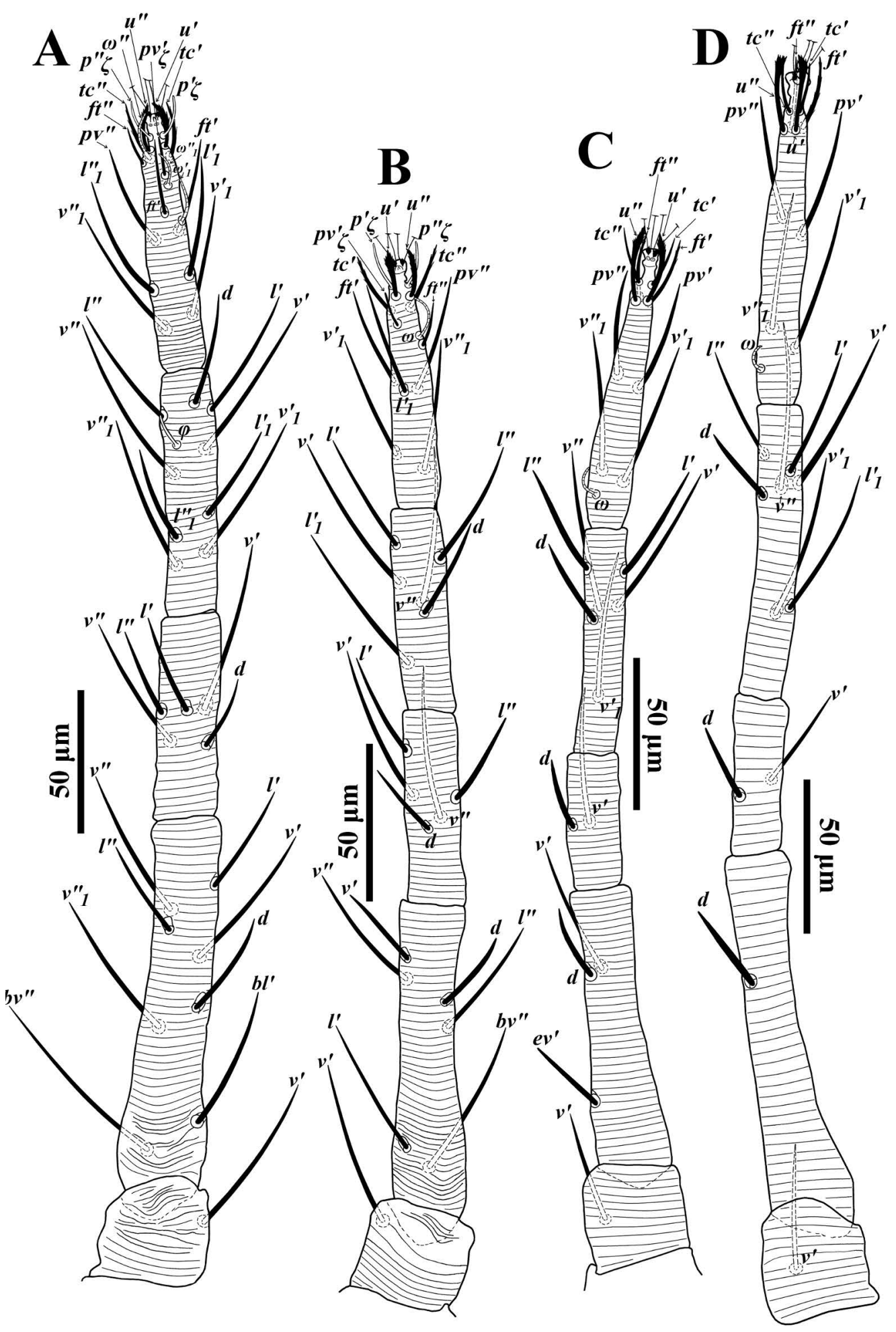

FIGURE 4: Eutetranychus orientalis (Klein) Female: A - Leg I; B - Leg II; C - Leg III; D - Leg IV. 
Legs - (Figs. 4A-D). Leg I 320-385; leg II 237345; leg III 276-349, leg IV 279-400. Legs long; leg I longer than body length. Leg segment setal formula as follows: coxae 2-1-1-1; trochanters 1-1-1-1; femora 8-6/7-3/4-1/2; genua 5-5-2-2; tibiae $9(1 \varphi)$-6-

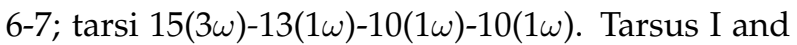
IV with solenidia $\mathrm{I} \omega^{\prime} 1$ 10-14, $\mathrm{I} \omega^{\prime \prime} 1$ 10-14, I $\omega^{\prime \prime} 11-16$, II $\omega$ 10-15, III $\omega$ 8-12, IV $\omega$ 6-11 and tibia I with $\mathrm{I} \varphi$ 1016.

Male (Figs. 5-6; $\mathrm{n}=95)-$ Color in life red. Idiosoma oval. Body length including gnathosoma 367425, excluding gnathosoma 225-307; width 205-242.

Dorsum (Fig. 5A) - Propodosoma with broken, longitudinal striae medially except anterior of sejugal furrow; opisthosoma with irregular striae medially except area anterior of setae $d 1$ with transverse striae, posterior opisthosoma with transverse striae, lateral idiosoma with longitudinal to oblique striae (Fig. 5A). Dorsal setae thick, subulate, more slender than female setae; dorsocentral setae $(c 1, d 1, e 1, f 1)$ shorter than other dorsal setae, excepting slender, sparsely serrate setae $h 2$ and $h 3$. Lengths of dorsal setae $v 2$ 31-40, sc1 35-48, sc2 26-38, c1 13-20, $c_{2} 29-41$, $c_{3}$ 29-37, d1 13-22, d2 26-37, e1 14-21, e2 25-32, f1 1523, f2 23-31, h1 20-26, h2 13-19, h3 13-20. Distances between dorsal setae; v2-h1 231-334, v2-v2 48-57, sc1sc1 93-115, sc2-sc2 159-189, c1-c1 36-50, d1-d1 73-82, e1-e1 36-46, f1-f1 30-39, h1-h1 18-28, c1-d1 37-53, d1e1 41-53, e1-f1 32-38, f1-h1 38-48.

Venter (Figs. 5B-C) - Ventral integument with transverse striae. Length of setae $1 a$ 40-47, 1b 4760, 1c 43-59, $2 b$ 44-53, 3a 34-50, 3b 43-54, 4a 38-47, $4 b$ 40-53, ag 21-30, g1 13-17, g2 13-17, ps1 8-12, ps 2 813. Distal bent portion of aedeagus shorter than dorsal margin of shaft, which is relatively straight (Fig. 5C).

Gnathosoma (Figs. 5B, D) - Ventral infracapitulum with two pairs of adoral setae or 16 (5-7) and or $_{2}$ 6 (5-6) and one subcapitular seta $m$ 31-37. Palp eupathidion $s u \xi$ of male shorter than that of female, length 3-4, width 1 , solenidion $\omega$ length 3 , width 1. Stylophore anteriorly rounded. Peritremes with simple bulb distally (Fig. 5A).

Legs (Figs. 6A-D) - Leg II 381-441; leg III 357-424, leg IV 370-454. Legs long; leg I longer than body length. Leg segment setal formula as follows: coxae 2-1-1-1; trochanters 1-1-1-1; femora 8-6-4-1/2; genua 5-5-2-2; tibiae $9(4 \varphi)-6(2 \varphi)-6(1 \varphi)$ -

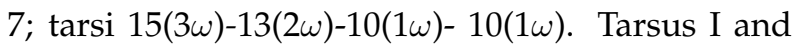
IV with solenidia $\mathrm{I} \omega 1^{\prime} 10-16$, I $\omega 1^{\prime \prime} 10-18$, I $\omega^{\prime \prime} 10-16$, II $\omega 1$ " 9-13, II $\omega "$ "10-15, III $\omega$ 10-13, IV $\omega$ 8-12 and tibia I with I $\varphi 1$ 11-15, I $\varphi 2$ 9-15, I $\varphi 3$ 10-17, I $\varphi 4$ 10-18, II $\varphi 1$ 11-15, II $\varphi 2$ 10-15, III $\varphi$ 8-14. Leg setae serrated (Figs. $6 \mathrm{~A}-\mathrm{D})$.

Deutonymph (Fig. 7; n=32) - Idiosoma oval. Body length including gnathosoma 272-322, excluding gnathosoma 205-250; width 195-220.

Dorsum (Fig. 7A) - Prodorsum with longitudinal striae, opisthosoma with transverse striae medially and longitudinal striae laterally (Fig. 7A). Dorsal setae on small tubercles and narrowly lanceolate; lengths of dorsal setae $v 2$ 37-57, sc1 40-64, sc2 40-61, c1 18-52, c 2 39-65, c $c_{3} 36-53, d 1$ 18-53, d2 44-62, e1 1846, e2 38-62, f1 36-54, f2 32-54, h1 28-38. Distances between dorsal setae: v2-h1 188-230, v2-v2 42-53, sc1sc1 84-107, sc2-sc2 129-188, c1-c1 37-53, d1-d1 68-87, e1-e1 33-50, f1-f1 24-37, h1-h1 14-25, c1-d1 25-42, d1e1 33-55, e1-f1 20-37, f1-h1 24-49.

Venter (Fig. 7B) - Ventral integument with transverse striae; ventral setae simple. Length of setae $1 a$ 22-32, $1 b$ 30-40, 1c 29-40, 2b 31-41, 3a 24-38, $3 b$ 20-38, $4 a$ 22-30, $4 b$ 25-31, ag 16-29, g 11-22, h2 11-17, h3 11-18, ps1 8-12, ps 2 8-12.

Gnathosoma (Fig. 7C) - Ventral infracapitulum with two pairs of adoral setae and subcapitular setae $m$ 23-38. Palp and peritremes similar to female and as indicated in figures 7C and 7A. Eupathidion $s u \xi$ length 6-8, width 1-2, solenidion $\omega$ length 2-3, width 1.

Legs (Figs. 7D-G) — Leg I 168-208; leg II 153-195; leg III 148-188, leg IV 140-195. Leg segment setal formula as follows: coxae 2-1-1-1; trochanters 1-1-10 ; femora 5-3-2-2; genua 5-5-2-1; tibiae $7(1 \varphi)-5-5-5$; tarsi $13(3 \omega)-11(1 \omega)-8-8$. Tarsus I and II with solenidia $\mathrm{I} \omega^{\prime} 14-10, \mathrm{I} \omega^{\prime \prime} 1$ 5-9, I $\omega^{\prime \prime} 7-12$, II $\omega$ 5-10 and tibia I with $\mathrm{I} \varphi$ 6-11.

Protonymph (Fig. 8; n=8) - Idiosoma oval. Body length including gnathosoma 237-295, excluding gnathosoma 177-230; width 165-210.

Dorsum (Fig. 8A) - Prodorsum with longitudinal striae and opisthosoma with transverse striae 

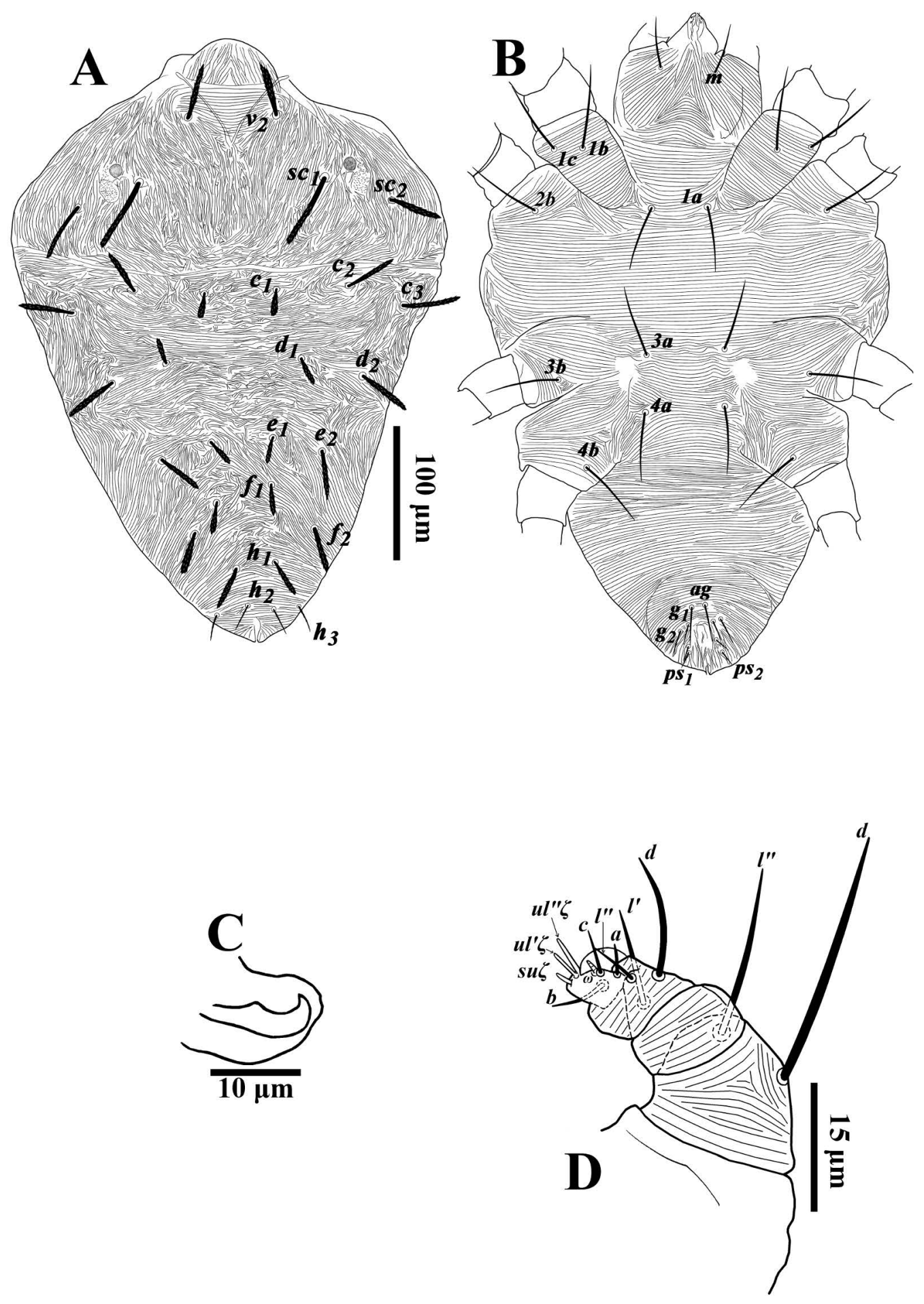

FIGURE 5: Eutetranychus orientalis (Klein) Male: A - Dorsal view; B - Ventral view; C - Aedeagus; D - Palp. 


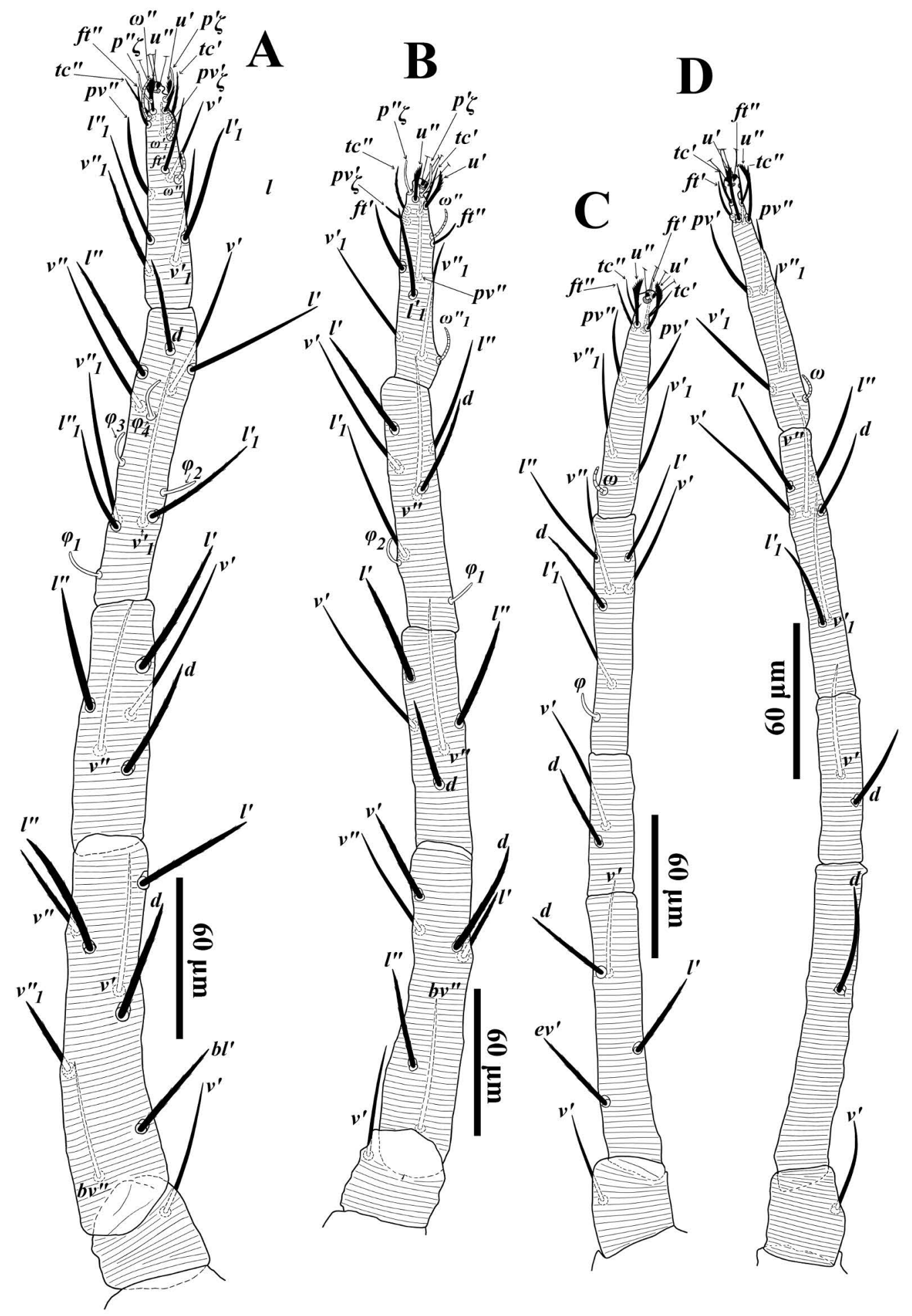

FIGURE 6: Eutetranychus orientalis (Klein) Male: A - Leg I; B - Leg II; C - Leg III; D - Leg IV. 


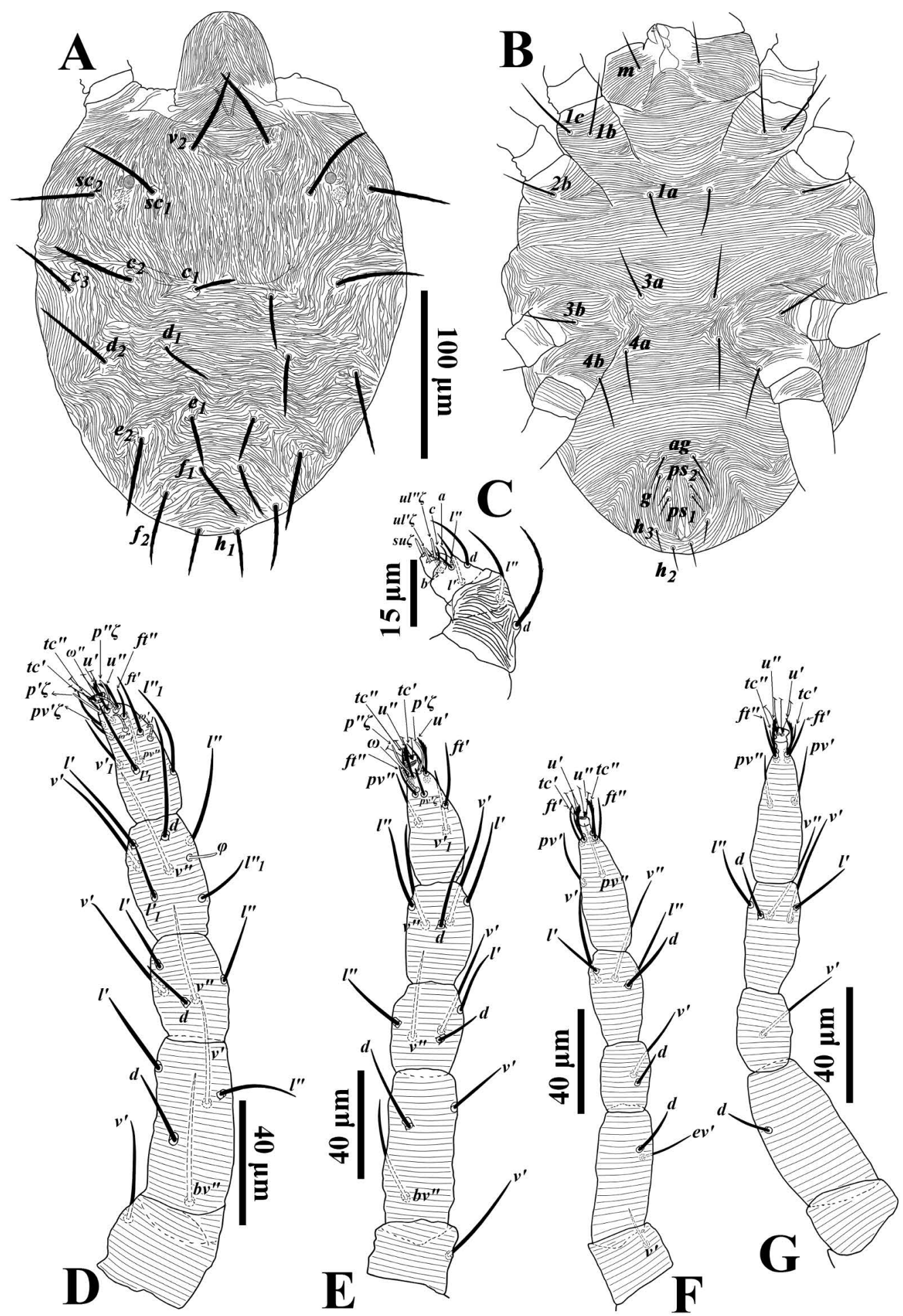

FIGURE 7: Eutetranychus orientalis (Klein) Deutonymph: A - Dorsal view; B - Ventral view, C - Palp; D - Leg I; E - Leg II; F - Leg III; G Leg IV. 


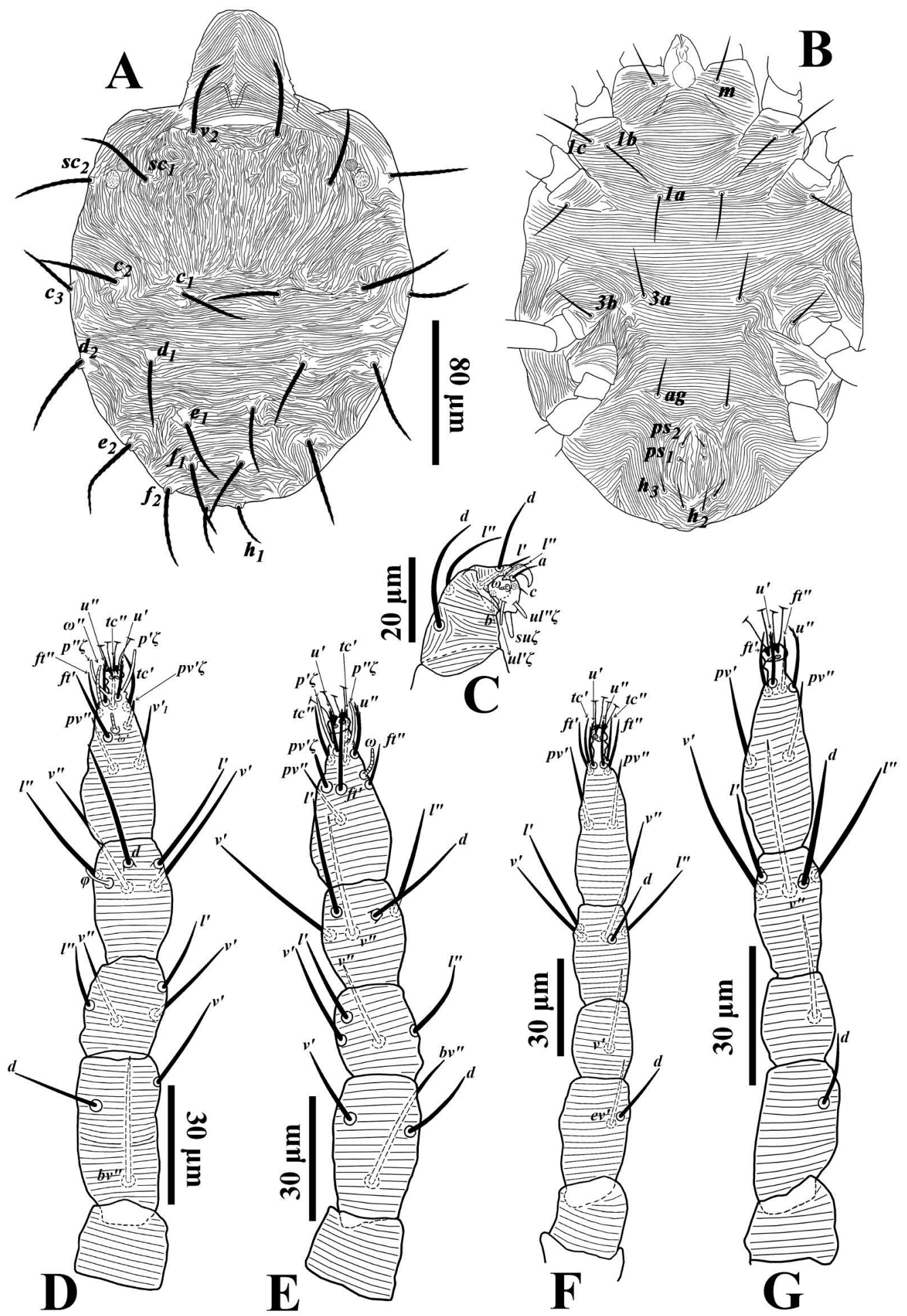

FIGURE 8: Eutetranychus orientalis (Klein) Protonymph: A - Dorsal view; B - Ventral view; C - Palp; D - Leg I; E - Leg II; F - Leg III; G Leg IV. 
(Fig. 8A); peritremes and dorsal setae similar to deutonymph. Lengths of dorsal setae $v 241-45$, sc1 4147, sc2 41-46, c1 33-39, c 2 45-53, c 3 34-42, d1 36-39, d2 43-50, e1 32-38, e2 38-52, f1 34-41, f2 38-45, h1 25-35. Distances between dorsal setae: v2-h1 165-223, v2v2 43-50, sc1-sc1 82-100, sc2-sc2 132-160, c1-c1 45-50, d1-d1 64-82, e1-e1 25-37, f1-f1 23-29, h1-h1 17-24, c1d1 20-53, d1-e1 26-46, e1-f1 19-29, f1-h1 22-30.

Venter (Fig. 8B) - Ventral pattern similar to deutonymph. Length of setae $1 a$ 22-30, $1 b$ 25-32, 1c 2630, 2b 23-28, 3a 21-33, 3b 21-27, ag 13-19, ps1 8-12, $p s_{2}$ 7-10, h2 9-14, h3 9-11.

Gnathosoma (Fig. 8C) - Ventral infracapitulum with setae $m$ 20-23. Palp similar to deutonymph and as indicated in figure 8C. Eupathidion $s u \xi$ length 67 , width 1 , solenidion $\omega$ length 2 , width 1 .

Legs (Figs. 8D-G) — Leg I 117-150; leg II 125-133; leg III 110-160, leg IV 93-127. Leg segment setal formula as: coxae 2-1-1-0; trochanters $0-0-0-0$; femora 3-

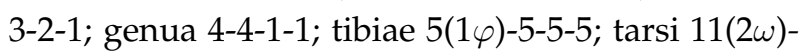
$10(1 \omega)-8-6$. Tarsi I and II with solenidia I $\omega^{\prime} 5-6, \mathrm{I} \omega^{\prime \prime}$ 6-10, II $\omega$ 6-8, and tibia I with $\mathrm{I} \varphi$ 6-9.

Larva (Fig. 9; $\mathrm{n}=2$ ) - Body length including gnathosoma 235, excluding gnathosoma 195; width 165.

Dorsum (Fig. 9A) - Prodorsum and opisthosoma with transverse striae; dorsal setae long and serrate (Fig. 9A). Lengths of dorsal setae: $v 2$ 37-43, sc1 39-42, sc2 30-38, c1 31-34, $c_{2} 42-51, c_{3} 32-37, d 1$ 3338, d2 37-42, e1 34-39, e2 41-46, f1 39-44, f2 38-41, h1 25-32. Distances between dorsal setae: v2-v2 43-50, sc1-sc1 85-90, sc2-sc2 112-122, c1-c1 41-50, d1-d1 5663, e1-e1 14-22, f1-f1 14-15, h1-h1 10-13, c1-d1 19-37, d1-e1 27-44, e1-f1 15-27, f1-h1 14-23.

Venter (Fig. 9B) - Ventral integument between setae $1 a-3 a$ with transverse striae and posterior venter with longitudinal to oblique striae (Fig. 43). Length of setae $1 a$ 19-22, $1 b$ 21-24, 3a 16-22, ps1 8-11, $p s_{2} 8-11, h 2$ 8-15, h3 15.

Gnathosoma (Fig. 9C) - Palp as indicated in figure 9C. Eupathidion $s u \xi$ length 7, width 1 , solenidion $\omega$ length 2 , width 1 .

Legs (Figs. 9D-F) — Leg I 108-115; leg II 96-100; leg III 99-107. Leg segment setal formula as follows: coxae 1-0-0; trochanters 0-0-0; femora 3-3-2; genua 44-1; tibiae 5(1 $\varphi)-5-5$; tarsi $8(1 \omega)-8(1 \omega)-6$. Tarsi I and II with solenidia I $\omega$ 8-10, II $\omega$ 6-7, and tibia I with I $\varphi$ 6-7.

Material examined. Iran - 156 females, 95 males, 32 deutonymphs (DN), 8 protonymphs ( $\mathrm{PN})$, 2 larvae (L) as follows. Kermanshah province: 4 females, 1 male ex. apricot leaves, Prunus armeniaca (Rosaceae), Dalahoo, Banmazaran village, $34^{\circ} 29^{\prime} \mathrm{N}$, $46^{\circ} 01^{\prime} \mathrm{E}, 1456$ m.a.s.l., 29 Oct. and 2 Nov. 2006, A. Babak Fard; 20 females, 4 males, 2 DN, 1 PN ex. fig leaves, Ficus carica (Moraceae), Dalahoo, Banmazaran village, $34^{\circ} 29^{\prime} \mathrm{N}, 46^{\circ} 01^{\prime} \mathrm{E}, 1456$ m.a.s.l., 23 Sep., 29 Oct. and 2 Nov. 2006, A. Babak Fard; 7 females, 1 male, $1 \mathrm{DN}$ walnut leaves, Juglans regia (Juglandaceae), Dalahoo, Banmazaran village, $34^{\circ} 29^{\prime} \mathrm{N}, 46^{\circ} 01^{\prime} \mathrm{E}, 1456$ m.a.s.l., 23 Sep., 29 Oct. and 2 Nov. 2006, A. Babak Fard; 6 females, 1 DN ex. peach leaves, Prunus domestica (Rosaceae), Dalahoo, Banmazaran village, $34^{\circ} 29^{\prime} \mathrm{N}, 46^{\circ} 01^{\prime} \mathrm{E}, 1456$ m.a.s.l., 2 Nov. 2006, A. Babak Fard; 4 females, 1 male, $2 \mathrm{DN}$ ex. black mulberry leaves, Morus nigra (Moraceae), Dalahoo, Banmazaran village, $34^{\circ} 29^{\prime} \mathrm{N}$, $46^{\circ} 01^{\prime}$ E, 1456 m.a.s.l., 2 Nov. 2006, A. Babak Fard; 1 female, 8 males ex. plum leaves, $P$. domestica, Dalahoo, Banmazaran village, $34^{\circ} 29^{\prime} \mathrm{N}, 46^{\circ} 01^{\prime} \mathrm{E}, 1456$ m.a.s.l., 2 Nov. 2006, A. Babak Fard; 1 male ex. almond leaves, Prunus dulcis (Rosaceae), Dalahoo, Banmazaran village, $34^{\circ} 29^{\prime} \mathrm{N}, 46^{\circ} 01^{\prime} \mathrm{E}, 1456$ m.a.s.l., 2 Nov. 2006, A. Babak Fard; 1 female ex. grapevine leaves, Vitis vinifera L. (Vitaceae), Dalahoo, Banmazaran village, $34^{\circ} 29^{\prime} \mathrm{N}, 46^{\circ} 01^{\prime} \mathrm{E}, 1456$ m.a.s.l., 23 Sep. 2006, A. Babak Fard; 1 female ex. quince leaves, Cydonia oblonga (Rosaceae), Dalahoo, Banmazaran village, $34^{\circ} 29^{\prime} \mathrm{N}, 46^{\circ} 01^{\prime} \mathrm{E}, 1456$ m.a.s.l., 2 Nov. 2006, A. Babak Fard; 7 females, 20 males, 3 DN, 1 PN ex. peach leaves, $P$. domestica, Dalahoo, Babajani village, $34^{\circ} 44^{\prime} \mathrm{N}, 46^{\circ} 09^{\prime} \mathrm{E}$, altitude $1250 \mathrm{~m}$ a.s.l., 23 Sep. 2006, A. Babak Fard; 4 females, 5 males, 7 DN ex. plum leaves, P. domestica, Dalahoo, Babajani village, $34^{\circ} 44^{\prime} \mathrm{N}, 46^{\circ} 09^{\prime} \mathrm{E}$, altitude 1250 m.a.s.l., 23 Sep. 2006, A. Babak Fard; 2 females, 1 DN, 1 PN ex. pomegranate leaves, Punica granatum (Lythraceae), Dalahoo, Babajani village, $34^{\circ} 44^{\prime} \mathrm{N}, 46^{\circ} 09^{\prime} \mathrm{E}$, altitude 1250 m a.s.l., 23 Sep. 2006, A. Babak Fard; 4 females, 9 males, $1 \mathrm{DN}$ ex. fig leaves, F. carica, Dalahoo, 

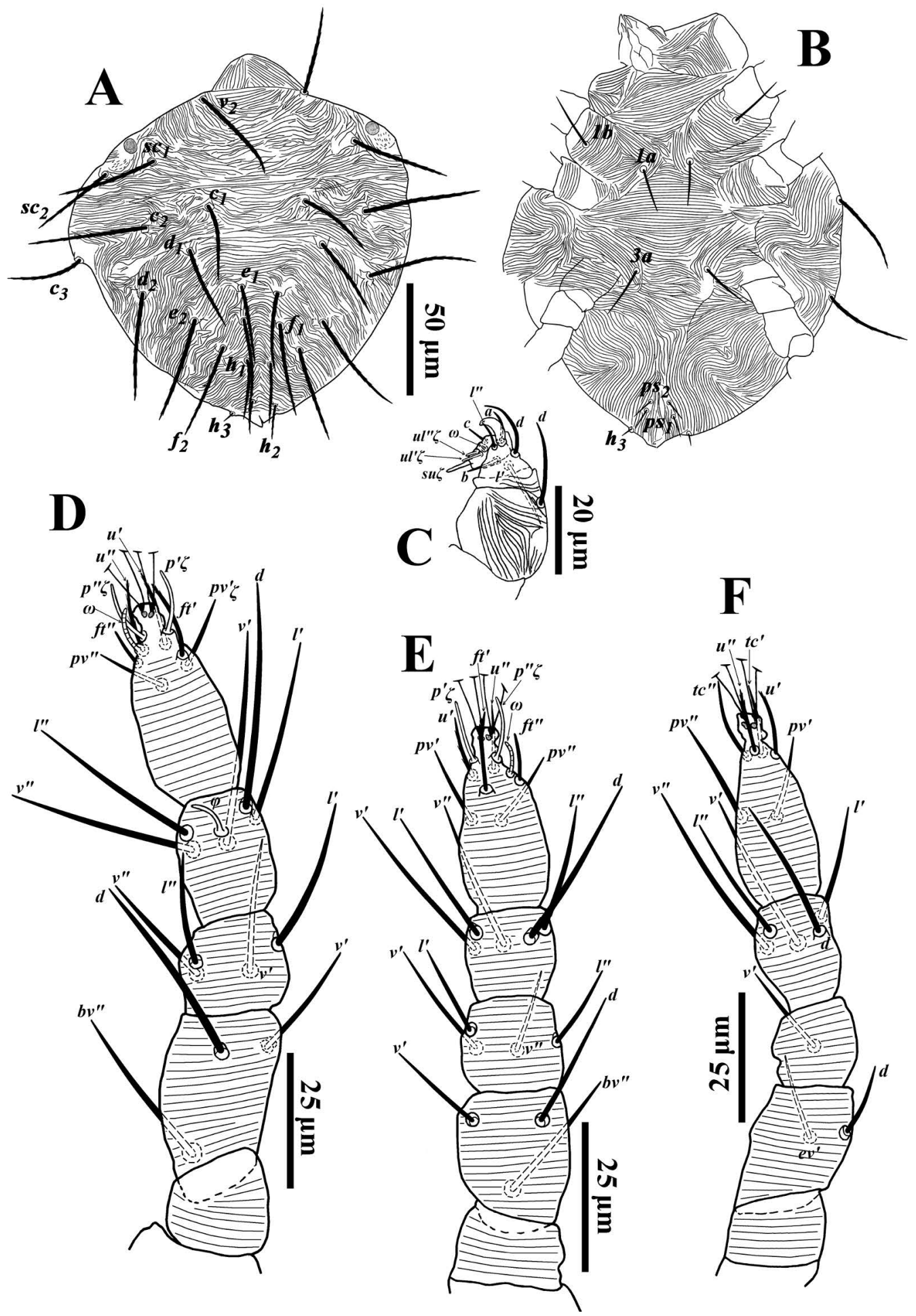

FIGURE 9: Eutetranychus orientalis (Klein) Larva: A - Dorsal view; B - Ventral view, C - Palp; D - Leg I; E - Leg II; F - Leg III. 
Babajani village, $34^{\circ} 44^{\prime} \mathrm{N}, 46^{\circ} 09^{\prime} \mathrm{E}$, altitude $1250 \mathrm{~m}$ a.s.l., 23 Sep. 2006, A. Babak Fard; 5 females, 3 males ex. walnut leaves, J. regia Dalahoo, Babajani village, $34^{\circ} 44^{\prime} \mathrm{N}, 46^{\circ} 09^{\prime} \mathrm{E}$, altitude 1250 m.a.s.l., 28 Oct. and 1 Nov. 2006, A. Babak Fard; 1 female ex. grapevine leaves, $V$. vinifera Dalahoo, Babajani village, $34^{\circ} 44^{\prime} \mathrm{N}, 46^{\circ} 09^{\prime} \mathrm{E}$, altitude 1250 m.a.s.l., 23 Sep. 2006, A. Babak Fard; 4 females, 2 DN, 1 PN ex. apricot leaves, P. armeniaca, Dalahoo, Babajani village, $34^{\circ} 44^{\prime} \mathrm{N}, 46^{\circ} 09^{\prime} \mathrm{E}$, altitude 1250 m.a.s.l., 2 Nov. 2006, A. Babak Fard; 8 females, 5 males, 2 PN ex. apple leaves, Malus domestica, Dalahoo, Babajani village, $34^{\circ} 44^{\prime} \mathrm{N}, 46^{\circ} 09^{\prime} \mathrm{E}$, altitude 1250 m.a.s.l., 23 Sep. 2006, A. Babak Fard; 2 females, 1 DN ex. fig leaves, F. carica, Dalahoo, Shalan village, $34^{\circ} 28^{\prime} \mathrm{N}, 45^{\circ} 59^{\prime} \mathrm{E}$, 1070 m.a.s.l., 29 Sep. 2006, A. Babak Fard; 3 females ex. black mulberry leaves, M. nigra, Dalahoo, Shalan village, $34^{\circ} 28^{\prime} \mathrm{N}, 4^{\circ} 59^{\prime} \mathrm{E}, 1070$ m.a.s.l., 29 Sep. 2006, A. Babak Fard; 1 female, 1 male ex. pomegranate leaves, P. granatum, Dalahoo, Shalan village, $34^{\circ} 28^{\prime} \mathrm{N}, 45^{\circ} 59^{\prime} \mathrm{E}, 1070$ m.a.s.l., 29 Sep. 2006 , A. Babak Fard; 1 female, 1 DN ex. pear leaves, Pyrus persica (Rosaceae), Dalahoo, Shalan village, $34^{\circ} 28^{\prime} \mathrm{N}$, $45^{\circ} 59^{\prime}$ E, 1070 m.a.s.l., 29 Sep. 2006, A. Babak Fard; 1 female, 1 male, $1 \mathrm{DN}, 1 \mathrm{PN}$ ex. plum leaves, P. domestica, Dalahoo, Shalan village, $34^{\circ} 28^{\prime} \mathrm{N}, 45^{\circ} 59^{\prime} \mathrm{E}, 1070$ m.a.s.l., 29 Sep. 2006, A. Babak Fard; 18 females, 9 males, 3 DN, 1 PN ex. walnut leaves, J. regia, Dalahoo, Shalan village, $34^{\circ} 28^{\prime} \mathrm{N}, 4^{\circ} 59^{\prime} \mathrm{E}, 1070$ m.a.s.l., 29 Sep. and 1 Nov. 2006, A. Babak Fard; 25 females, 2 males, 3 DN ex. apricot leaves, P. armeniaca, Dalahoo, Shalan village, $34^{\circ} 28^{\prime} \mathrm{N}, 4^{\circ} 59^{\prime} \mathrm{E}, 1070$ m.a.s.l., 29 Sep., 19 Oct. 2006, A. Babak Fard, 1 Nov. 2011, A. Azizi; 3 females, 5 males ex. peach leaves, P. domestica, Dalahoo, Shalan village, $34^{\circ} 28^{\prime} \mathrm{N}, 45^{\circ} 59^{\prime} \mathrm{E}$, 1070 m.a.s.l., 29 Sep. and 1 Nov. 2006, A. Babak Fard; 1 male ex. grapevine leaves, V. vinifera, Dalahoo, Shalan village, $34^{\circ} 28^{\prime} \mathrm{N}, 4^{\circ} 59^{\prime} \mathrm{E}, 1070$ m.a.s.l., 29 Sep. 2006, A. Babak Fard; 1 female, 1 male ex. walnut leaves, J. regia, Dalahoo, Zhalkeh village, $34^{\circ} 34^{\prime} \mathrm{N}, 46^{\circ} 41^{\prime} \mathrm{E}, 1121$ m.a.s.l., 25 Sep. 2006, A. Babak Fard; 8 females, 12 males, ex. peach leaves, $P$.

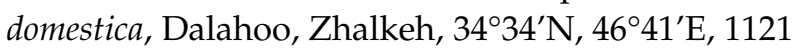
m.a.s.l., 25 Sep. 2006, A. Babak Fard; 1 male, 1 DN ex. fig leaves, F. carica, Dalahoo, $34^{\circ} 31^{\prime} \mathrm{N}, 47^{\circ} 08^{\prime} \mathrm{E}$, 1428 m.a.s.l., 25 Sep. 2006, A. Babak Fard; 1 female, 1 male, $2 \mathrm{DN}$ ex. grapevine leaves, $V$. vinifera
L., Dalahoo, $34^{\circ} 31^{\prime} \mathrm{N}, 47^{\circ} 08^{\prime} \mathrm{E}, 1428$ m.a.s.l., 25 Sep. 2006, A. Babak Fard. Other provinces: 4 females, $1 \mathrm{DN}, 1 \mathrm{~L}$ ex. sweet orange leaves, Citrus sinensis (L.) Osbeck (Rutaceae), Kreman province, Bam, $29^{\circ} 06^{\prime} \mathrm{N}, 58^{\circ} 21^{\prime} \mathrm{E}, 1050$ m.a.s.l., 9 Oct. 2009, A. Asad Abadi; 2 females, ex. plum leaves, $P$. domestica, Kurdistan province, Dehgolan, $35^{\circ} 27^{\prime} \mathrm{N}, 47^{\circ} 41^{\prime} \mathrm{E}, 1906$ m.a.s.l., 15 Oct. 2006, V. Safasadati; 2 females, 1 L, ex. lemon leaves, Citrus limon (L.) Osbeck, Fars province, Shiraz, $29^{\circ} 39^{\prime} \mathrm{N}, 52^{\circ} 39^{\prime} \mathrm{E}, 1486$ m.a.s.l., 27 Nov. 2014, S. Bakhshi. Deposited in the Collection of the Acarology Laboratory, University of Bu-Ali Sina, Hamadan, Iran, excepting 2 females, 1 male, 1 deutonymph, 1 protonymph and 1 larva to be deposited in Queensland Museum, South Brisbane, Australia.

Australian specimens (not in redescription): 26 females, 19 males, as follows. Queensland: 2 females, 2 males ex. red cedar in glasshouse, Toona ciliata (Meliaceae), Townsville, $19^{\circ} 15^{\prime} 52^{\prime \prime} S, 146^{\circ} 48^{\prime} 55^{\prime \prime} \mathrm{E}$, 31 May 1994, M. Webb; 1 female, 1 male ex. ornamental tree, University of Queensland, St Lucia, 22 Mar. 1996, D.E. Walter; 3 females, 2 males ex. Erythrina haerdii (Fabaceae), Mt Coot-tha Botanical Gar-

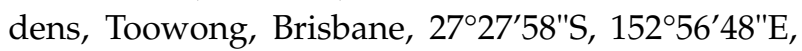
7 May 2000, S. Ozman; 2 females, 2 males ex. Albizia lebbeck (Mimosaceae), Mt Larcom, $67 \mathrm{~km} \mathrm{~S}$ Rockhampton, $23^{\circ} 48^{\prime} 45^{\prime \prime}$, $150^{\circ} 58^{\prime} 44^{\prime \prime} \mathrm{E}$; 2 females, 2 males ex. Murraya ovatifoliata (Rutaceae) [plant moved from Koolkoorum Ck nr Miriamvale, SE Queensland], Brisbane, The Gap, 54 Barkala St, $27^{\circ} 26^{\prime} \mathrm{S} 152^{\circ} 57^{\prime} \mathrm{E}, 8$ May 2005, J.J. Beard; 4 females, 2 males ex Murraya paniculata, Coorparoo, Stanley St East, $27^{\circ} 29^{\prime} \mathrm{S}, 153^{\circ} 02^{\prime} \mathrm{E}, 18$ Mar. 2004, J.J. Beard; 2 females, 2 males ex. frangipani Plumeria acutifolia

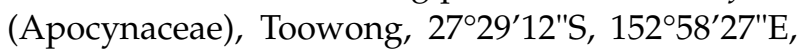
4 Apr 2000, S. Ozman. 1 female, 1 male ex. Carica papaya (Caricaceae), Torres Strait, Horn Is., 2 Jul. 2011, L. Halling; 1 female, 1 male ex. frangipani, Plumeria sp., Torres Strait, Coconut Island, 13 Mar 2007, A.D. Rice; 1 female ex. Glycine max (Fabaceae). Northern Territory: 4 females, 2 males ex. Manihot esculenta (Euphorbiaceae), Galiwinku Island, 1201'49"S,

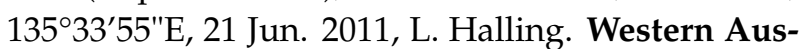
tralia: Kununurra, 21 Sep. 2009, L. Halling; 2 fe-

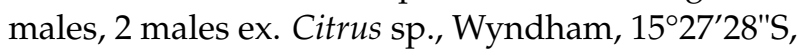


128 06'12"E, 16 May 2012, L. Halling; 1 female ex Heliconia sp. (Heliconiaceae), Broome, 15 Sep. 2011, L. Halling.

Iranian variant (not in redescription): 6 females as follows. 5 females ex. soil under palm trees, Phoenix dactylifera (Arecaceae), Kerman province, Bam, $29^{\circ} 06^{\prime} \mathrm{N}, 58^{\circ} 21^{\prime} \mathrm{E}, 1050$ m.a.s.1., 20 Apr. and 10 May 2010, A. Asad-Abadi; 1 female ex. soil under sweet orange tree, Citrus $\times$ sinensis (Rutaceae), Kerman province, Bam, $29^{\circ} 06^{\prime} \mathrm{N}, 58^{\circ} 21^{\prime} \mathrm{E}$, 1050 m.a.s.1., 11 Apr. 2010, A. Asad-Abadi; 5 females deposited as slide-mounted specimens in the Collection of the Acarology Laboratory, University of Bu-Ali Sina, Hamadan, Iran; 1 female will be deposited in Queensland Museum, South Brisbane, Australia.

Remarks - The species definition of Eut. orientalis presents one of the most difficult problems in spider mite taxonomy, with few rivals, such as debate surrounding Tetranychus urticae Koch and its synonyms, especially Tetranychus cinnabarinus Dufour. While that debate is largely resolved thanks to a greater research effort into the problem (Xie et al. 2008; Mendonça et al. 2011; Auger et al. 2013), the difficulties surrounding Eut. orientalis have not received similar research effort, probably because it is a less important pest species. The problem may be more complex, because Eut. orientalis shows more intraspecific variation than species of Tetranychus. Due to this variation the species already has four synonyms (Anychus ricini, Eut. anneckei, Eut. monody, Eut. sudanicus) and we suspect several more exist, pending examination of types. In the absence of molecular evidence of a cryptic species complex, we think it best to consider a broad morphological concept for this species.

The most striking intraspecific variation is in the form of the setae, which vary in length and in shape (subulate, narrowly to broadly lanceolate, spatulate, ovate and obovate). This variation was reported in Baker \& Pritchard (1960), who provided the first synonymy (Eut. ricini), and Chaudhri et al. (1974) provided a series of drawings showing remarkable variation. As noted by Baker \& Pritchard (1960), and confirmed by later observation and personal observation of specimens, they can vary within popu- lations and also asymmetrically on the same specimen. Despite this variation, some generalisations can be made. The setae are always heavily barbed, so much as to be plumose, and the dorsocentral setae $(c 1, d 1, e 1)$ are often small, and are always smaller than the lateral setae and sublateral seta c2. The dorsocentral setae are usually lanceolate, ovate or obovate; while the lateral setae are subulate (thick but tapering) to spatulate (i.e. long, with lanceolate tips). We have not yet found specimens with dorsocentral setae as long as those shown in Chaudhri et al. (1974), but considering the variation we have observed, such variations are not unexpected.

Gutierrez \& Helle (1971) conducted crossing experiments and suggested that Eut. orientalis represented a species complex, yet future work has been unable to prove a species complex exists. Meyer (1974) concurred with Baker \& Pritchard (1960) that setal length and form should be dismissed as a useful character to separate species within Eut. orientalis, but did consider the pattern of striae between d1-e1 diagnostic, and also the length of the terminal palpal sensillum $s u \xi$ relative to the solenidion $\omega$. On this basis, Meyer (1974) established the species Eut. anneckei. However, Meyer (1987) broadened the species concept for Eut. orientalis and rejected the striation pattern between $d 1-e 1$ as diagnostic because this state varied within populations, being Ushaped, V-shaped or entirely longitudinal. Thus, she synonymized her species Eut. anneckei with Eut. orientalis and also considered Eut. monodi and Eut. sudanicus as synonyms of Eut. orientalis.

Meyer's (1987) broader diagnosis comprised two states not included in our diagnosis above: the palp tarsus eupathidion $s u \xi$, equivalent to the spinneret in web-spinning spider mites, is about three times as long as wide, and setae $c 1, c 2$ and $c 3$ in a transverse line. Each of these character states are subject to significant variation and are not used in our diagnosis. First, the palp sensillum was indirectly considered to vary by Meyer (1987) when she synonymized the species Eut. anneckei, Eut. monodi and Eut. sudanicus. Our studies have shown similar variation, with the sensillum about 3-4 times longer than wide. Second, setae $c 1, c 2$ and $c 3$ do 
not form a perfect line, with $c 2$ often anterior of that line, and the character state is likely used to distinguish species with setae $c 2$ anterior and lateral, such as Eut. cratis Baker \& Pritchard.

Leg setae are also used to define the species, but these vary intraspecifically in Eut. orientalis. To explore leg setal variation in Eut. orientalis, we examined the setal counts on the femur, genu and tibia of 26 female specimens from Australia and 50 specimens from Iran. These leg segments are often used to distinguish species. For the Australian specimens, only two had the typical setal count of femora 8-6-3-1; genua 5-5-2-2; tibiae $9(1 \varphi)-6-6-7$. Of the remaining 24 , there was one setal difference in 12 specimens, two differences in 7 specimens and three differences in 5 specimens. These differences were mostly asymmetrical (32 of 39 anomalies), with the typical number of setae always expressed on the other side. In the cases of symmetrical differences, these were additional setae on femur III $(\mathrm{n}=2)$ and tibia II $(\mathrm{n}=1)$ and the absence of a seta on femur I $(\mathrm{n}=1)$; the other three symmetrical differences were the expression of three solenidia on tibia I $(n=1)$ and two solenidia on tibia II $(n=2)$. Another two specimens expressed solenidia asymmetrically: one with $3 / 4 \varphi$ on tibia I (partial male condition) and one with $0 / 1 \varphi$ on tibia II (partial male condition). Differences in tactile setal counts are summarized as follows: femur I with 8/9 setae $(\mathrm{n}=1), 7$ setae $(\mathrm{n}$ $=1)$, femur II with $6 / 7$ setae $(\mathrm{n}=1)$, femur III with $3 / 4$ setae $(n=11), 2 / 3$ setae $(n=1), 4$ setae $(n=2)$, femur IV with $1 / 2$ setae $(\mathrm{n}=2)$; tibia I with $9 / 10$ setae $(\mathrm{n}=3)$; tibia II with $6 / 7$ setae $(\mathrm{n}=2), 7$ setae $(\mathrm{n}=$ $1)$; tibia III with $6 / 7$ setae $(\mathrm{n}=1)$; tibia IV with $7 / 8$ setae $(n=3)$.

The Iranian specimens also showed considerable variation, but much less so that the Australian specimens. Of the 50 specimens, 24 had the typical setal count. Of the remaining 26 , there was one setal difference in 21 specimens, two differences in 2 specimens and three differences in 3 specimens. These differences were mostly asymmetrical. Differences in tactile setal counts are summarized as follows: femur I with $7 / 8$ setae $(\mathrm{n}=1)$, femur II with $6 / 7$ setae $(n=2)$, femur III with $3 / 4$ setae $(n=7), 4$ setae $(n=8)$, femur IV with $1 / 2$ setae $(n=3), 2$ setae $(n=2)$; genu
II with $4 / 5$ setae $(n=5)$; tibia III with 5 setae $(n=1)$, tibia IV with $6 / 7$ setae $(n=5)$.

Overall, the genual setal counts were stable, with just one specimen with an additional seta on genu III. In contrast, the other leg segments were much more variable. This data shows that variation should be expected on the femora and tibiae of Eut. orientalis, especially femur III, which accounted for almost one third of variation. We stress that leg setation remains useful in defining species of Eutetranychus, but several individuals from different populations should be examined to establish species definitions. Species of Eutetranychus described from few individuals, or just one collection, should be treated with skepticism.

Initially, we considered the possibility that the Iranian specimens of Eut. orientalis found on date palm represented a different species, as they differed in spermathecal shape (spermatheca pointed, instead of rounded in other Iranian specimens and in other descriptions), presence of pores between $d 2$ $e 2$, aedeagal shape (subtle differences in shape), and palp tarsal sensillum, presence of pores between d2-e2, aedeagal shape (subtle differences in shape), and palp tarsal sensillum dimensions. However, examination of Australian Eut. orientalis showed that the spermatheca can be pointed or rounded, and the pores are always present in Eutetranychus, and probably all spider mites; they are simply not drawn in many descriptions. The aedeagus is subject to some variation, which may be intraspecific or - more likely - varies according to focal point and orientation during slide mounting, as shown for Tetranychus in Seeman \& Beard (2011). We note that species of Eutetranychus differ little in aedeagal morphology, unlike some other spider mite genera, and most species have a thick, upward-curving prong. Finally, as noted above, the dimensions of the palp sensillum are subject to some variation, and only striking and consistent differences in this character state should be used to define species.

The specimens collected from date palm presented a more compelling reason to consider these a new species. These five females were the only specimens collected from date palm, and unlike the numerous other specimens collected from other plants, 
TABLE 1: Range of selected measurements for Eutetranychus orientalis on date palm, other Iranian specimens, and Australian specimens. Measurements in bold highlight the larger range of either the specimens from date palm or the other Iranian specimens. Measurements for the Australian specimens are given for comparison.

\begin{tabular}{|c|c|c|c|c|c|c|c|}
\hline Character & date palm & Iran & Australia & Character & date palm & Iran & Australia \\
\hline$v_{2}-h_{1}$ & $257-315$ & $287-369$ & $250-355$ & $1 c$ & $45-52$ & $49-58$ & $38-58$ \\
\hline$S C_{1}$ & $26-34$ & $33-57$ & $29-55$ & $2 b$ & $38-48$ & $46-56$ & $42-50$ \\
\hline$S C_{2}$ & $27-39$ & $41-54$ & $24-43$ & $3 a$ & $38-45$ & $45-52$ & $32-42$ \\
\hline$c_{2}$ & $23-35$ & $40-58$ & $25-55$ & $3 b$ & $40-46$ & $47-60$ & $32-50$ \\
\hline$c_{3}$ & $25-37$ & $42-51$ & $26-40$ & $4 a$ & $38-47$ & $42-54$ & $30-49$ \\
\hline$d_{2}$ & $28-36$ & $39-58$ & $25-57$ & $4 b$ & $44-50$ & $46-58$ & $31-51$ \\
\hline$e_{2}$ & $29-40$ & $37-54$ & $25-53$ & $a g$ & $35-43$ & $42-56$ & $27-47$ \\
\hline$v_{2}-v_{2}$ & $60-76$ & $49-60$ & $42-56$ & $g_{1}$ & $27-33$ & 31-39 & $24-32$ \\
\hline$S C_{1}-S C_{1}$ & 122-135 & $109-128$ & $100-145$ & $g_{2}$ & $24-32$ & $30-35$ & $24-35$ \\
\hline$c_{1}-c_{1}$ & $57-75$ & $48-62$ & 51-63 & $p s_{1}$ & $13-20$ & $10-16$ & $10-15$ \\
\hline$d_{1}-d_{1}$ & 87-105 & 98-122 & $90-110$ & $p s_{2}$ & $14-20$ & $11-16$ & $10-14$ \\
\hline $1 b$ & $41-52$ & 47-57 & $34-51$ & $m$ & $30-39$ & $38-52$ & $26-40$ \\
\hline
\end{tabular}

these specimens had a different dorsal strial pattern, being more U-shaped, and seven setae on femur II. However, as shown by Meyer (1987), differences in strial patterns constitute intraspecific variation in Eut. orientalis. Furthermore, examination of variation demonstrated that femur II had variable leg setation, including one specimen with seven setae expressed symmetrically (six is the usual number). Although all five specimens had seven leg setae, expressed symmetrically, it is possible these specimens represented a small founding population where seven leg setae had become fixed. The specimens also differed in several measurements, but the ranges of measurements were not completely exclusive of those of Eut. orientalis (Table 1). Generally, the body was shorter, as were several body setae, especially on the venter. The tendency for smaller dimensions, and absence of immature stages, may suggest these mites were a small population struggling to exist on a non-preferred host plant, although Chaudhri et al. (1974) also recorded the species from date palm. The Australian specimens also had overlapping ranges with both the $E$ utetranychus from palms and those from other host plants in Iran. Finally, we note that there was no variation in the number of coxal setae in any specimens, which is an important character state that separates Eutetranychus africanus (Tucker) from Eut. orientalis.

\section{Tribe Eurytetranychini Reck Genus Eurytetranychus Oudemans}

Eurytetranychus Oudemans, 1931:224.

Anychus McGregor, 1919: 644.

Type species: Tetranychus latus Oudemans (= Neotetranychus buxi Garman)

Diagnosis - Modified from Meyer (1987). Dorsal opisthosoma with ten pairs of setae (c1-3, d12 , e1-2, f1-2, h1), venter with setae $h 2-3$; dorsal setae may be inserted on tubercles; peritremes simple or slightly expanded distally; anal valves with two pairs of anal setae (ps1-2); tarsi I and II with or without a pair of 'associated setae' (bases of $f t$ " and $\omega^{\prime \prime}$ touching but not coalesced), true claws padlike, with tenent hairs, empodium small and clawlike but distinct.

Remarks - Eurytetranychus spp. are closely related to Eutetranychus, differing only in the size of the empodium, which is small and claw-like in Eurytetranychus but absent or vestigial in Eutetranychus. Meyer (1987) diagnosed the genus as having a pair of associated setae on tarsus I, which are homologous to the duplex setae, i.e., the anterior fastigial seta $\mathrm{ft}^{\prime}$ and solenidion $\omega^{\prime}$. However, species of Eurytetranychus differ in how closely this seta and solenidion associate, and in some species they are clearly separate. 


\section{Eurytetranychus aminii n. sp. (Figures 10-11)}

Zoobank: 553B2463-BA28-4D9E-BB48-A92F814D97D3

Diagnosis - Prodorsum with folded transverse striae medially and longitudinal striae laterally; dorsal setae narrowly lanceolate; dorsocentral setae ( $c 1$, $d 1, e 1$ and f1) as long as or longer than longitudinal intervals between them. Leg segment setal for-

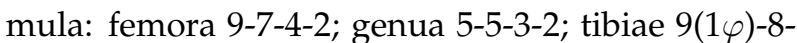

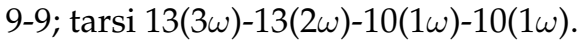

Description - Female (Figs. 10-11; n= 4) - Idiosoma broad oval. Body length including gnathosoma 545 (527-552), excluding gnathosoma 362 (382402); width 370 (357).

Dorsum (Figs. 10A-B) - Prodorsum with folded transverse striae medially and longitudinal striae laterally; opisthosoma with transverse striae medially and longitudinal striae laterally (Fig. 10A). Dorsal setae barbed, thickened, subulate, inserted on small tubercles (Fig. 10B). Lengths of dorsal setae $v 257$ (58-63), sc1 68 (65), sc2 54 (50-57), c1 64 (6166), $c_{2} 64$ (70), $c_{3} 50$ (52-64), d1 67, d2 64, e1 70 (70), e2 64, f1 70 (72), f2 61(65), h1 54 (57). Distances between dorsal setae; v2-h1 358 (357-380), v2-v2 50 (4755), sc1-sc1 100 (98-102), sc2-sc2 255 (235), c1-c1 75 (75-78), d1-d1 102 (101-107), e1-e1 69 (63-70), f1-f1 46 (40-55), h1-h1 49 (45-46), c1-d1 46 (45-57), d1-e1 55 (50-63), e1-f1 64 (55-70), f1-h1 75 (66-85).

Venter (Figs. 10C-D) - Area between setae 1aag with transverse striae; opisthosoma laterad genitoanal region with longitudinal to oblique striae (Fig. 10C). Length of setae la 49 (43-54), $1 b 61$ (5562), 1c 54 (58-63), $2 b 53$ (54-62), 2c 50 (49-60), $3 a 48$ (49-53), $3 b 50$ (49-54), $4 a 54$ (52-59), $4 b 53$ (50-54), ag 46 (52-57), g1 40 (40-42), g2 36 (38-43), ps1 16 (16-19), $p s_{2} 16$ (16-17), h2 36 (33-35), h3 28 (31-38).

Gnathosoma (Fig. 10E) — Ventral infracapitulum with two pairs of adoral setae, or 16 (5-7), or 26 (5-6), and one pair of subcapitular setae $m 41$ (45-50). Palp eupathidion $s u \xi$ length 7 (6-7), width 2(1-2), solenidion $\omega$ length 3(2-3), width 2(1-2). Peritremes linear, terminating in bulb.

Legs (Figs. 11A-E) — Leg I 264 (257-263); leg II 233 (218-225); leg III 236 (235-238); leg IV 256 (258265). Legs shorter than body. Leg segment setal formula as follows: coxae 2-2-1-1; trochanters 1-1-

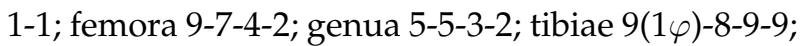

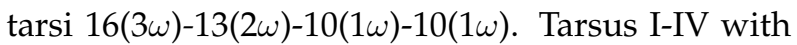
solenidia $\mathrm{I} \omega^{\prime} 16$ (5-7), I $\omega^{\prime \prime} 16$ (6-7), I $\omega^{\prime \prime} 13$ (11-14), II $\omega^{\prime \prime} 6$ (4-7), II $\omega^{\prime} 5$ (5-8), III $\omega 6$ (4-8), IV $\omega 5$ (5-7) and tibia I with I $\varphi 9$ (8-11). Leg setae weakly barbed (Figs. 11A-D). Tarsus I and II without associated setae. True claws pad-like, with tenent hairs, empodium small and clawlike (Fig. 11E).

Material examined - Holotype female and 3 female paratypes ex. gum bushes, Astragalus gossypinus Fisch. (Fabaceae), Iran: Kurdistan province,

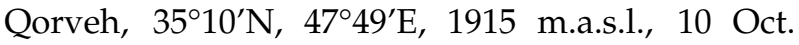
2013, F. Amini. The holotype female, two female paratypes are deposited as slide-mounted specimens in the Collection of the Acarology Laboratory, University of Bu-Ali Sina, Hamadan, Iran and two paratypes female will be deposited in Queensland Museum, South Brisbane, Australia.

Etymology - This species is named for Mrs. Fatemeh Amini, who collected the type specimens of the species.

Remarks - Many species of Eurytetranychus are poorly described and lack diagnostic information, such as dorsal striation patterns. The better descriptions of Pritchard \& Baker (1955), Gutierrez (1966), Meyer (1974, 1987) and Ehara (1980) are certainly adequate, but we note that the venter of a species of Eurytetranychus is illustrated here for the first time, even though Eur. aminii n. sp. is the $19^{\text {th }}$ species of this genus. Therefore, our decision to create a new species is affected by the quality of prior descriptions, and the two main distinctive character states of Eur. aminii n. sp. - the prodorsal striae and associated setae on tarsus I - could not be assessed completely.

Eurytetranychus aminii n. sp. lacks associated setae because solenidion $\omega^{\prime}$ and setae $f t^{\prime}$ are wellseparated from each other. Likewise, the descriptions of Eur. admes Pritchard \& Baker and Eur. wuyishanensis $\mathrm{Hu} \&$ Chen show that these species do not have these setae associated (Pritchard \& Baker 1955; $\mathrm{Hu} \&$ Chen 1994). In contrast, $\omega^{\prime}$ and $\mathrm{ft}^{\prime}$ are associated in the descriptions of Eur. buxi, Eur. cyclohalanopsis $\mathrm{Hu}$ \& Chen, Eur. neobuxi Meyer and Eur. spathatus Meyer (Pritchard \& Baker 1955; Meyer 

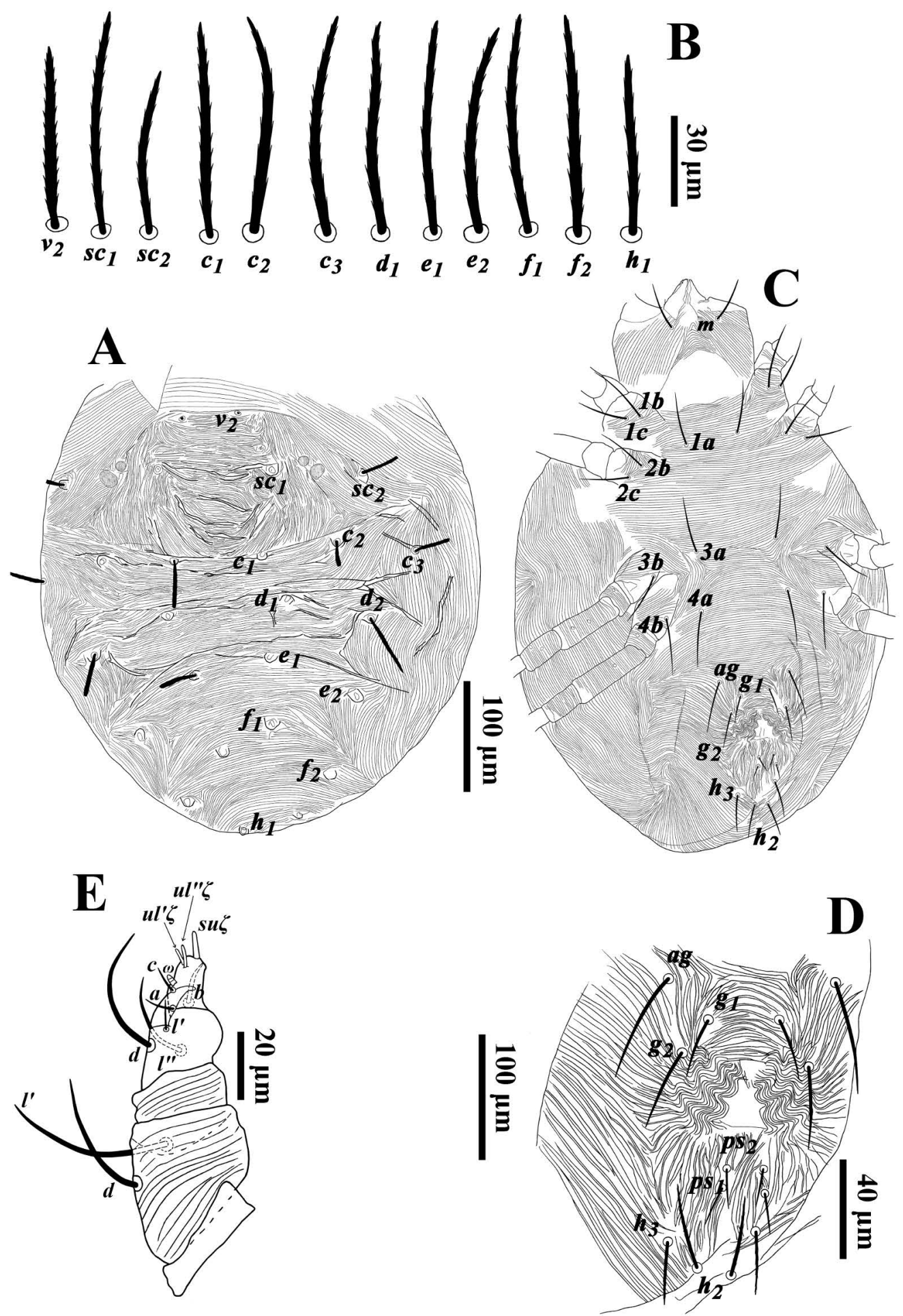

FIGURE 10: Eurytetranychus aminii n. sp. Female: A - Dorsal view; B - Dorsal setae; C - Ventral view; D - Genitoanal area; E - Palp. 


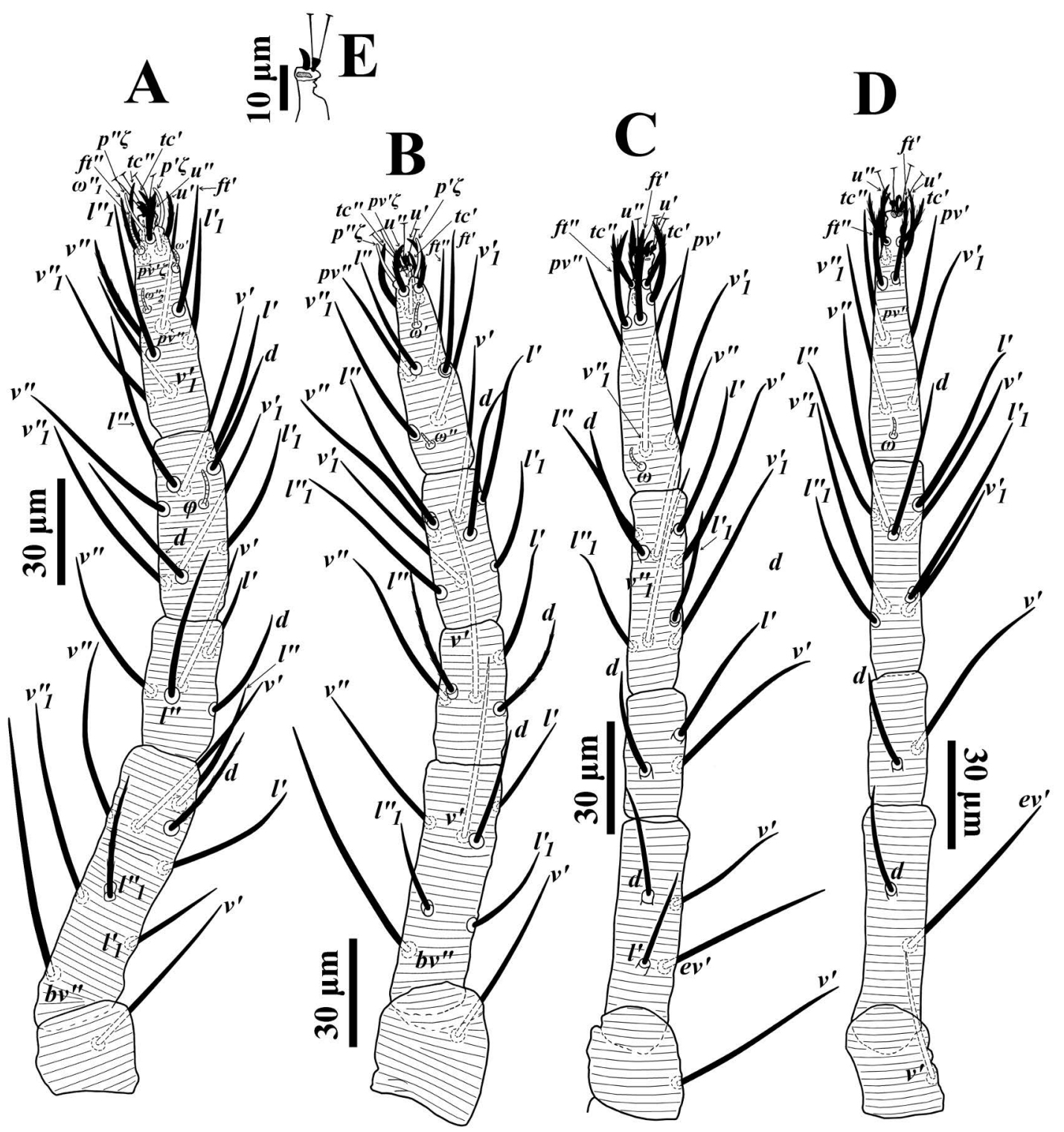

FIGURE 11: Eurytetranychus aminii n. sp. Female: A - Leg I; B - Leg II; C - Leg III; D - Leg IV; E - Empodium and claws. 
1974; Hu \& Chen 1994). These setae are also probably associated in Eur. acacia Meyer and Eur. madagascariensis Gutierrez because Meyer (1987) examined these species and her diagnosis for Eurytetranychus includes the presence of associated setae on tarsus I.

Previous work on Eurytetranychus used the form and size of body setae, and leg setal counts, to distinguish species. Few other diagnostic states, if any, are used within the genus. As pointed out in the case of Eut. orientalis (see above, and also Gutierrez (1977) and Meyer (1987)), tetranychid mites are not always morphologically conservative, especially in body setae shape and size, and leg setal counts. Eurytetranychus is no exception, as Eur. neobuxi Meyer varies in leg setal counts (Meyer 1987). Indeed, polymorphic dorsal setae and leg setal variation may be widespread in the Eurytetranychini and Aponychini. For example, Hernandes and Feres (2013) provided evidence of intraspecific variation in the Aponychini, suggesting numerous synonyms exist in the group. We conclude the same is likely in $E \mathcal{~ - ~}$ rytetranychus and Eutetranychus.

In addition to polymorphism in dorsal setal form and intraspecific variation in leg setal counts, we must also rely upon the accuracy of past descriptions in the absence of easily-accessed type specimens or quality data on these types. However, despite our reservations, we describe this new species because it of its unusual transverse central prodorsal striae, lack of associated setae, the fact that it cannot be easily accommodated within any other species of Eurytetranychus, occurs on a novel host for the Eurytetranychini (Migeon \& Dorkeld 20062015), and also provides morphological data hitherto absent for this genus.

As mentioned above, the new species does not match any of the reasonably well-described species of Eurytetranychus due to the transverse central prodorsal striae. Of the remaining species, Eur. aminii n. sp. resembles E. huaqingnicus $\mathrm{Ma}$ and Yuan and E. fengchengensis Ma and Yuan by sharing tapering dorsal setae, setae $d 1$ and $e 1$ longer than the longitudinal intervals between them and femur III with four setae (Ma \& Yuan 1981). However E. aminii $\mathbf{n}$. sp. differs from E. huaqingnicus by: tib- iae II and III with nine setae in new species whereas seven setae in E. huaqingnicus; femur IV with two setae in E. aminii versus three setae in E. huaqingnicus; and setae $f 11.25$ times longer than $h 1$ in new species instead of four times longer in E. huaqingnicus.

Eurytetranychus aminii $\mathbf{n}$. sp. differs from the $E$. fengchengensis by: setae $c 1$ longer (setae $c 1$ 0.8-0.9 as long as distance between setae $c 1-c 1$ ) in the new species but shorter (setae $c 1$ ca. 0.5 distance $c 1-c 1$ ) in E. fengchengensis; tarsi II and IV with 15 and 11 setae respectively in new species but 14 and 10 setae in E. fengchengensis; and tibiae II and III with nine setae in new species whereas with seven setae in $E$. fengchengensis.

The morphological variation present within this group, coupled with substandard species descriptions, compels us to urge caution with the use of the key below. Within Eurytetranychus, we also suggest several "species groups" that may instead be groups of synonymous species, pending examination of type specimens. These are numbered as follows, with a comment on the likelihood of these species being synonyms in square brackets. Each group is based on the form and size of the dorsal setae only as this is shown in most descriptions, but this makes the assumption that dorsal setae are not polymorphic. While polymorphic setae occur in some other Eurytetranychini, they are not yet known for Eurytetranychus, so these groups may be of some help in identifying species.

Group 1 - Dorsocentral setae $(c 1, d 1, e 1, f 1)$ long and slender, extending beyond bases of next row of setae. Eurytetranychus ulmi (on Ulmus sp.).

Group 2 - Dorsal setae long and slender, extending beyond bases of next row of setae, except setae c1, shorter. Eur. fengchengensis, Eur. huaqingnicus [on Ulmus sp.] based on only 2-3 females; very high chance of being synonyms of Eur. ulmi].

Group 3 - Dorsocentral setae $(c 1, d 1, e 1)$ short (setae length $c 1<d 1<e 1$ ), less than two times shorter than dorsolateral setae and $h 1$, setae $f 1$ the longest. Dorsal setae lanceolate and serrate, not inserted on tubercle with the exception of setae $f 1, f 2$ and $h 1$ set on tubercle. Eur. shenyangensis and Eur. acaciae.

Group 4 - Dorsocentral setae $(c 1, d 1, e 1)$ short (setae length $c 1<d 1<e 1$ ) and more than two times 
shorter than dorsolateral setae. Dorsal setae slender and serrate set on tubercles. Eur. koreanus.

Group 5 - Dorsal setae of moderate length, most or all opisthosomal extending about half-way or more than half-way to next row of setae. Eur. glycyrrhizae, Eur. aminii n. sp., Eur. buxi, Eur. madagascariensis, Eur. neobuxi, Eur. recki. [various host plants; Eur. recki with thickened setae; Eur. madagascariensis and Eur. neobuxi are differentiated by small differences in dorsal setal length and palpal sensillar size, so are a chance of being synonyms].

Group 6 - Dorsal setae very small, slender. Eur. mexicanus on Cupresus sp. and Eur. admes, Eur. furcisetus, Eur. piceus on Piceus spp. These species (Eur. admes, Eur. furcisetus, Eur. piceus) have a high chance of being synonyms, especially Eur. furcisetus and Eur. piceus, which are European. Unfortunately, the description of Eur. piceus is baffling in its lack of detail of even the most basic character states, but detailed examination of insignificant parts of the mite's morphology].

Group 7 - Dorsal setae of different length, lanceolate and serrate, lateral setae and setae f1 long, dorsocentral setae short and setae $d 1$ the shortest. Eur. cyclobalanopsis [on Quercus glauca].

Group 8 - Dorsal setae of different lengths and forms, lateral setae and $f 1, h 1$ long and lanceolate, dorsocentral setae $(c 1, d 1, e 1)$ and $s c 1, c 2$ short and spatulate. Eur. wuyishanensis.

Group 9 - All dorsal setae very small and spatulate. Eur. spathatus.

\section{ACKNOWLEDGEMENTS}

The authors wish to thank Prof. Dr. Carlos Flechtmann, Departamento de Entolomogia e Acarologia (LEA) (ESALQ), University of São Paulo, São Paulo, Brazil, for supplying literature. This article is a part of the $\mathrm{Ph}$. D thesis program in Agricultural Entomology and was supported by the University of Bu-Ali Sina, Iran. The authors thank the vice presidency for research of Bu-Ali Sina University for financial support of this project.

\section{REFERENCES}

André M. 1954 - Tétranyque nouveau, parasite de Cassia siamea Lam. et Grewia mollis Juss. à Dakar. Bul. de l'Institut Français d'Afrique Noire (série A), 16: 359361.

Attiah H.H. 1967 - The genus Eutetranychus in U.A.R., with description of three new species - Bull. Soc. Entomol. Egypte, 51: 11-16.

Auger P., Migeon A., Ueckermann E.A., Tiedt L., Navajas M. 2013 - Evidence for synonymy between Tetranychus urticae and Tetranychus cinnabarinus (Acari, Prostigmata, Tetranychidae): Review and new data Acarologia, 43: 383-415.

Bagdasarian A.T. 1948 - Novyj vid nautinnogo klesca iz Armenii - Dokl. Akademia Nauk Armenia SSR. Erevan, 9: 129-130.

Bagdasarian A.T. 1954 - New species of tetranychid mites from Armenia - Dokl. Akademia Nauk Armenia SSR. Erevan, 18: 51-56.

Baker E.W. and Pritchard, A.E. 1960 - The tetranychoid mites of Africa - Hilgardia, 29: 455-574.

Baker E.W., Tuttle D.M. 1994 - A guide to the spider mites (Tetranychidae) of the United States - Indira Publishing House, West Bloomfield, USA, 347 pp.

Banks N. 1917 - New Mites, mostly economic (Arach., Acar.) — Entomol. News, 28: 193-199.

Berlese A. 1910 - Lista di nuove specie e nuove generi di acari - Redia, 6: 242-271.

Beyzavi G., Ueckermann E.A., Faraji F., Ostovan H. 2013 - A catalog of Iranian prostigmatic mites of superfamilies Raphignathoidea \& Tetranychoidea (Acari — P. J. Acarol., 2(3): 89-474.

Bolland H.R., Gutierrez J. \& Flechtmann C.H.W. 1998 - World catalogue of the spider mite family (Acari: Tetranychidae) - Brill, Leiden, 392 pp.

Chaudhri W.M., Akbar S., Rasool A. 1974 - Taxonomic studies of the mites belonging to the families Tenuipalpidae, Tetranychidae, Tuckerellidae, Caligonellidae, Stigmaeidae and Phytoseiidae - University of Agriculture, Lyallpur, Pakistan Project No. A17-Ent., 26: 1-250.

Donnadieu A.L. 1875 - Recherches pour servir a l'histoire des Tetranyques - These. Faculte des Sciences de Lyon. 134 pp. Also published in: Annales de la Société Linnéenne de Lyon. (n. ser.), 22: 1876, 29-136.

Ehara S. 1995 - A new species of Tetranychus (Acari, Tetranychidae) from the Ryukyu Islands - Jpn. J. Entomol., 63: 229-233.

Elbadry E.A. 1970 - A new species of tetranychid mite from Sudan (Acarina: Tetranychidae) - Rev. Zool. Bot. afr., 82: 301-305. 
Estebanes-Gonzalez M.L., Baker E.W. 1968 - Ara-as rojas de Mexico (Acarina: Tetranychidae) - An. Esc. Nac. Cienc. Biol., 15: 61-133.

Flechtmann C.H.W. 1997 — Mites (Arthropoda: Acari) associated of palms (Arecaceae) in Brazil. III. Eutetranychus nomurai n. sp. (Tetranychidae) - Int. J. Acarol., 23: 269-273. doi:10.1080/01647959708683576

Gutierrez J. 1966 - Cinq nouvelles espèces de Tetranychidae de Madagascar - Acarologia, 8: 594-610.

Gutierrez J., Helle W. 1971 — Deux nouvelles espèces du genre Eutetranychus Banks (Acariens: Tetranychidae) vivant sur plantes cultivées à Madagascar - Entomol. Ber., Amst., 31: 45-60.

Gutierrez J. 1985 - Division of the Tetranychidae into sub-families, tribes, and genera. In: Helle, W. \& Sabelis, M.W. (Eds.), Spider Mites. Their biology, natural enemies and control - World Crop Pests 1A, 82-90.

Hernandes F.A., Feres R.J.F. 2013 - Phylogeny and taxonomic revision of the spider mite genera Aponychus, Paraponychus and Stylophoronychus using morphology (Acari: Tetranychidae) - Invertebr. Syst., 27: 265-281. doi:10.1071/IS12071

Hu S.Q., Chen X.W. 1994 - Two new species of the genus Eurytetranychus from China (Acari: Tetranychidae) Acta Zootaxonomica Sinica, 19: 302-305.

Jeppson L.R., Keifer H.H., Baker E.W. 1975 — Mites Injurious to Economic Plants - Univ. Calif. Press, Berkeley, 614 pp. +74 plates.

Khanjani M., Kamali K. 1998 - A study of Acari fauna of Tetranychoidea in Hamedan - 13th Iranian Plant Protection Congress, Karaj, Iran, p. 215.

Klein H.Z. 1936 - Contributions to the knowledge of the red spiders in Palestine. I. the Oriental red spider, Anychus orientalis Zacher. II. The common red spider, Epitetranychus althea v. Hainstein - Bull. Israel Agric. Res. Stn. Rehovot, 21, 3-36, 37-63.

Lindquist E.E. 1985 - Chapter 1.1 Anatomy, Phylogeny and Systematics. In: Helle, W. \& Sabelis, M.W. (Eds.), Spider Mites. Their biology, natural enemies and control Elsevier, Amsterdam, pp. 3-28.

Loyttyniemi K. 1973 - Eurytetranychus piceus sp.n. (Acarina, Tetranychidae) with notes on its life history Ann. Entomol. Fenn., 39: 119-123.

Ma E.P., Yuan Y.L. 1981 - Two new species of the genus Eurytetranychus from China (Acarina: Tetranychidae) —Zoological Research, 2: 195-198.

Ma E.P., Yuan Y.L. 1982 - A new genus and five new species of Tetranychidae from China (Acari: Tetranychidae) — Entomotaxonomia, 4: 109-114.

McGregor E.A. 1914 - Four new tetranychids Ann. Entomol. Soc. Am., 7: 354-364. doi:10.1093/aesa/7.4.354
Mendonça R.S. de, Navia D., Diniz I.R., Auger P., Navajas M. 2011 - A critical review on some closely related species of Tetranychus sensu stricto (Acari: Tetranychidae) in the public DNA sequences databases - Exp. Appl. Acarol., 55: 1-23. doi:10.1007/s10493-011-94535

Meyer M.K.P.S. 1974 - A revision of the Tetranychidae of Africa (Acari) with a key to the genera of the world - Entomology Memoir, Department of Agricultural Technical Services, Republic of South Africa, 36: 1-291.

Meyer M.K.P.S. 1987 - African Tetranychidae (Acari: Prostigmata) with reference to the world genera - Entomology Memoir. Department of Agriculture and Water Supply. Republic of South Africa, 69: 1-175.

Meyer M.K.P. (Smith), Ueckermann E. A. 1988 - South African Acari. III. On the mites of the Mountain Zel National Park — Koedoe, 31(1): 1-24.

Migeon A., Dorkeld F. 2006-2015 - Spider Mites Web: a comprehensive database for the Tetranychidae http:/ / www.montpellier.inra.fr/CBGP/spmweb

Miller L.W. 1966 - The tetranychid mites of Tasmania Papers and Proceedings of the Royal Society of Tasmania, 100: 53-76.

Nassar O.A., Ghai S. 1981 - Taxonomic studies on tetranychoid mites infesting vegetable and fruit crops in Delhi and surrounding areas - Orient. Insects, 15: 333-396. doi:10.1080/00305316.1981.10434337

Pritchard A.E., Baker E.W. 1955 - A revision of the spider mite family Tetranychidae - Memoirs Series, Pacific Coast Entomological Society, 2: 472.

Rahman K.A., Sapra A.N. 1940 - Mites of the family Tetranychidae from Lyallpur with descriptions of four new species. Indian Acad. Sci., Ser. B, 11: 17-196.

Reck G.F. 1950 - Spider mite fauna from Georgia (Tetranychidae: Acarina) - Trudy Zoologicheskogo Instituta Akademia Nauk Gruz.S.S.R., 9: 117-134.

Ries D.T. 1935 - A new mite (Neotetranychus buxi n. sp. Garman) on boxwood - J. Econ. Entomol., 28: 55-62. doi:10.1093/jee/28.1.55

Seeman O.D., Beard J.J. 2011 — Identification of exotic pest and Australian native and naturalised species of Tetranychus (Acari: Tetranychidae) - Zootaxa, 2961: 172.

Smiley R.L., Baker E.W. 1995 - A report on some tetranychid mites (Acari: Prostigmata) from Yemen - Int. J. Acarol., 21(3): 135-164. doi:10.1080/01647959508684055

Toroitich F., Ueckermann E.W., Thern P.D., Knapp M. 2009 - The tetranychid mites (Acari: Tetranychidae) of Kenya and a redescription of the species Peltanobia erasmusi Meyer (Acari: Tetranychidae) based on males - Zootaxa, 2176: 33-47. 
Tucker R.W.E. 1926 — Some South African mites, mainly Tetranychidae and Eriophyidae - South African Department of Agriculture Division of Entomology Memories, 5: 1-15.

Tuttle D.M., Baker E.W. 1968 - Spider mites of Southwestern United States and a revision of the family Tetranychidae - Tucson, Ariz. University of Arizona Press, 1-143.

Tuttle D.M., Baker E.W., Abbatiello M.J. 1976 - Spider mites of Mexico (Acari: Tetranychidae) - Int. J. Acarol., 2: 1-102. doi:10.1080/01647957608683760

Wainstein B.A. 1956 - Material on the fauna of tetranychid mites of Kazakhstan - Trudy respublik. Stantsii Zashchity Rastenii Kazifilial Vashnil, 3: 70-83. (In Russian).

Wainstein B.A. 1960 - Tetranychoid mites of Kazakhstan (with revision of the family) - Trudy Nauchno-Issled. Inst. Zashchita Rastenii Kazakh., 5: 1-276.

Wang H.F. 1977 - Eurytetranychus from China with a new species (Acarina: Tetranychidae) - Acta Entomologica Sinica, 20: 339-341.

Xie L.X, Xie R-R, Zhang K-J, Hong X-Y. 2008 - Genetic relationship between the Carmine Spider Mite Tetranychus cinnabarinus (Boisduval) and the Two-spotted Mite T. urticae Koch in China based on the mtDNA COI and rDNA ITS2 sequences - Zootaxa, 1726: 1832.

Yin S.G., Lu C.J., Lan W.H. 1987 - A new species of genus Eurytetranychus from China (Acarina : Tetranychidae) - Acta Entomologica Sinica, 30: 109-112

\section{COPYRIGHT}

(oc) EY-No-ND Khanjani M. et al. Acarologia is under free license. This open-access article is distributed under the terms of the Creative Commons-BY-NC-ND which permits unrestricted non-commercial use, distribution, and reproduction in any medium, provided the original author and source are credited. 